\title{
Les laboratoires de la Défense nationale
}

\author{
J. BRIAND-CHAMPLONG, P. LAROCHE ET M. GUILLAND
}

\section{Introduction}

Comme toutes les grandes entités employant des personnels travaillant sous rayonnements ionisants, le ministère de la Défense a été confronté au problème de leur surveillance dosimétrique et cela depuis sa mise en place durant la décennie 1950-1960. Cependant, la diversité des organismes qui composent le ministère de la Défense, la multiplicité des différents travaux sous rayonnements que l'on y rencontre et bien entendu la problématique particulière de la prise en compte non seulement du temps de paix mais aussi d'un éventuel temps de guerre, font que la dosimétrie dans la Défense présente une spécificité bien particulière.

En effet, la période de la Guerre froide et la menace d'un possible conflit avec utilisation de la force nucléaire a conduit à développer des dosimètres «guerre ». Les retombées (bénéfiques) de ces premières recherches du pharmacien général Chassende-Baroz du Service de santé des armées, considéré comme le père de la dosimétrie photographique, conduiront à des dosimètres plus généralistes utilisés par tous les laboratoires de dosimétrie. En effet, tous ces dosimètres sont le résultat d'une collaboration entre le Service de santé des armées, le Commissariat à l'énergie atomique et aussi des industriels comme la société Kodak.

Durant ces 50 dernières années, la surveillance dosimétrique et son organisation n'ont cessé d'évoluer au sein du ministère de la Défense suivant la progression des techniques de mesure et surtout en s'adaptant à la considérable avancée de l'informatique.

Compte tenu de leurs implications différentes dans les travaux sous rayonnements, plusieurs organismes de la Défense assuraient la dosimétrie de leurs personnels. L'évolution des programmes et les réorganisations internes ont bien entendu conduit à une uniformisation.

Les regroupements successifs des différents services internes chargés de la surveillance ont abouti à un seul laboratoire (Établissement technique de l'armement) qui gérait de plus la tenue du fichier général.

Enfin ce régime de croisière a débouché sur le transfert de cette surveillance au Service de protection radiologique des armées (SPRA) du Service de santé des 
armées (SSA) en 2000 et une organisation générale stabilisée avec l'accréditation du laboratoire en 2004 et l'intégration dans le système général français de surveillance de l'exposition des personnels tel que prévu par le code du Travail.

\subsection{Les différents dosimètres utilisés}

On distinguera successivement les dosimètres adaptés pour les rayonnements électromagnétiques X et gamma permettant dans certaines conditions de fournir des informations sur l'exposition aux rayonnements béta et les dosimètres destinés à la mesure des neutrons.

\subsubsection{Les dosimètres photographiques Chassende Baroz)}

Le pharmacien général Chassende-Baroz (figure 9.1), chercheur du Service de santé des armées, est à l'origine de la dosimétrie photographique de la Défense.

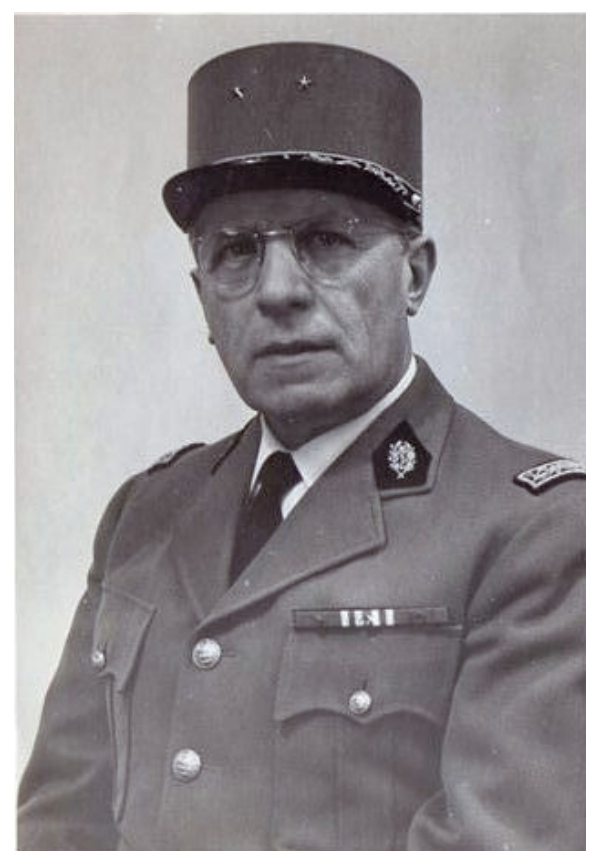

Figure 9.1 - Le pharmacien général Chassende-Baroz.

En 1954 - Le dosimètre guerre «FER 701 »

Il obtient son premier brevet sur un dosimètre photographique qu'il présente au Congrès de Genève en 1955 (figure 9.2). Ce dosimètre se présente comme un film 
dentaire de même format et avec le même type d'enveloppe étanche. Il enregistre des doses de 10 à 600 rads à l'aide de deux émulsions de sensibilité différente, sous filtre de plomb unique placé dans la pochette de conditionnement. Son interprétation se fait à l'aide d'une échelle de noircissement étalonnée. La mesure du noircissement est une mesure par réflexion.

Sa particularité est une bande témoin (raies noires et grises) qui prouve que le dosimètre a été correctement développé ; cette bande permet en lumière rouge inactinique de développer un film sans se soucier du temps, de la température et du vieillissement du bain révélateur. Ce procédé unique restera utilisé jusqu'au début des années 1980.

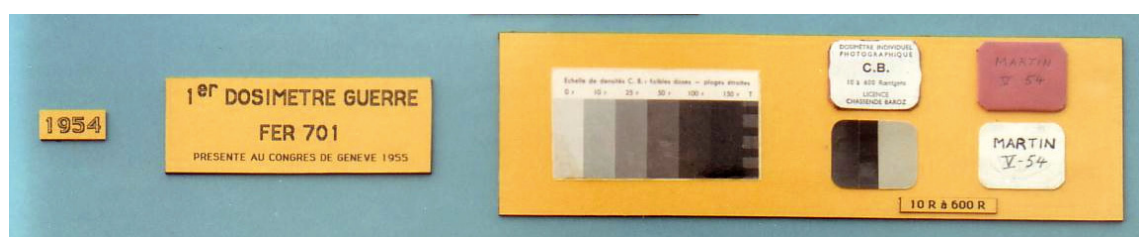

Figure 9.2 - Dosimètre Guerre 1954.

\section{En 1957 - Le dosimètre guerre «FER 701 - FER 702»}

Ce dosimètre, toujours de format dentaire, est conditionné sous pochette aluminium-polyéthylène qui le met à l'abri des vapeurs actiniques (mercure) et de l'humidité.

Un boîtier de plastique possédant un écran $1 \mathrm{~mm}$ de plomb est ajouté pour constituer le dosimètre «FER 702 ». Il permet d'enregistrer des doses de 10 à 800 rads (norme internationale) à l'aide de trois émulsions de sensibilités différentes. La mesure se fait de manière identique à celle du FER 701. Il est approuvé par la Direction du service de santé qui s'en approvisionne en réserve de guerre (figure 9.3).

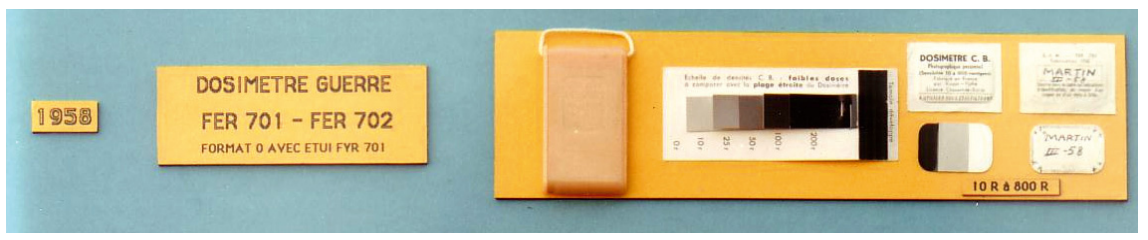

Figure 9.3-Dosimètre Guerre «FER 701-702 » 1958. 
Parallèlement, était conçu un kit de campagne permettant le développement des dosimètres sur le terrain (figure 9.4).

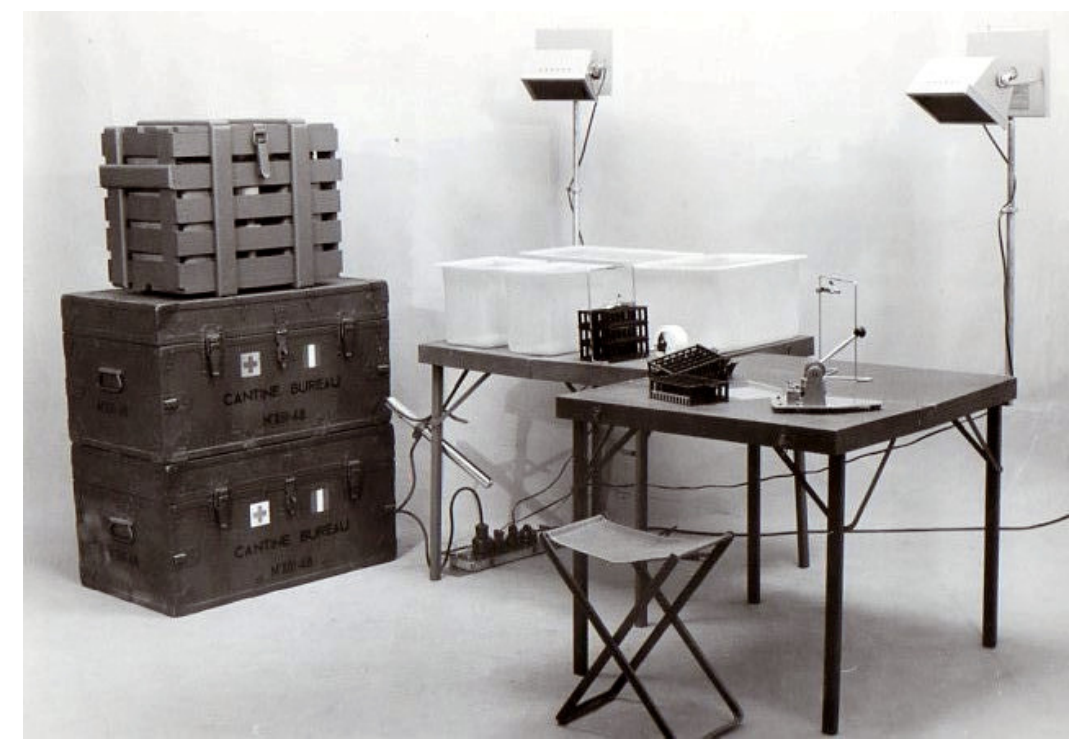

Figure 9.4 - Kit de campagne pour développer les dosimètres « guerre » sur le terrain.

Afin de s'assurer de la conservation de ces stocks, chaque année un prélèvement était effectué et les dosimètres développés et interprétés après irradiation à des valeurs connues.

On vérifiait également l'évolution du voile de fond des dosimètres (évolution de l'émulsion la plus sensible).

Après la chute du mur de Berlin, le spectre de la Guerre froide s'éloignant, la conservation de ces stocks fut abandonnée. Parallèlement, le développement de dosimètres électroniques type « défense » fut réalisé pour équiper les troupes en théâtres d'opérations. Par curiosité, nous avions conservé quelques dosimètres ; un dernier développement fut effectué en 2000 et il montra que seule la première émulsion était saturée.

À côté de ces dosimètres spécifiques et à partir de 1958, la dosimétrie de surveillance du personnel exposé aux rayonnements ionisants en temps de paix a été développée par le Service de santé des armées. 
Le dosimètre «DMA-X » a été mis en service au laboratoire de dosimétrie de l'Établissement central d'électroradiologie des armées (ECERA) pour la surveillance des personnels des hôpitaux et infirmeries militaires travaillant en radiodiagnostic.

Le premier dosimètre de radiodiagnostic basse énergie (figure 9.5) était de format 0 (dentaire enfant), conditionné sous pochette étanche de même type que le FER 702, et placé pour en faciliter le port dans un étui équipé de filtres plomb et cuivre type «Chassende- Baroz». Il permettait l'enregistrement des doses de 10 millirads à 10 rads, toujours avec une mesure par réflexion de la densité optique.

Le dosimètre «DMA-Co », premier dosimètre à haute énergie (figure 9.5), a été mis en service fin 1959 au laboratoire de dosimétrie de l'ECERA pour la surveillance des personnels manipulant du matériel émettant des radiations de haute énergie (Service de cobaltothérapie du Val de Grâce en particulier). Il était de format identique à celui du DMA X, enregistrait les doses de 20 millirads à 20 rads et placé dans un étui modifié pour être adapté aux rayonnements gamma de haute énergie.

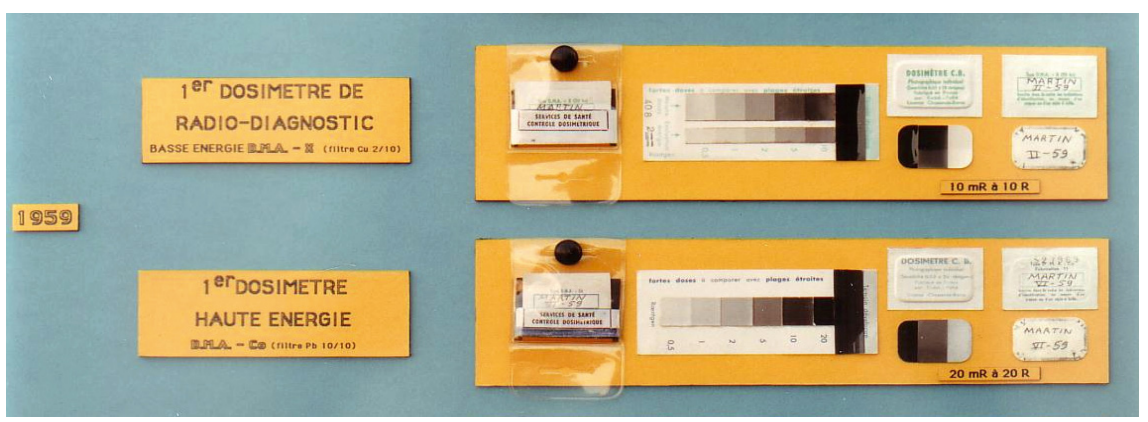

Figure 9.5 - Dosimètres pour le service de santé 1959.

L'évolution de ces dosimètres aboutit en 1960 au dosimètre « DMA-CEA » type 1 .

Ce dosimètre et son étui «PS1» sont nés de l'étroite collaboration entre l'ECERA, le CEA et la société Kodak. Il fut présenté au Congrès de Madrid de 1963 (voir chapitre 8). Sa plage de mesure permet de couvrir les doses de 20 millirads à 800 rads à l'aide de trois émulsions de sensibilité différente couchées recto verso sur un support central cellulosique. La mesure du noircissement reste une mesure par réflexion. La bande témoin de développement toujours présente assure le contrôle de la qualité du développement. 
Quant à l'étui «PS1 » il est composé de plusieurs filtres (aluminium, cuivre de diverses épaisseurs, cadmium, plomb), ce qui permet de déterminer l'énergie des rayonnements mesurés et l'interprétation des doses issues de ces différents rayonnements (gamma, X, béta) La mesure sous cadmium signale la présence de neutrons thermiques (information qualitative plus que quantitative).

Un « détrompeur » sous forme d'une bille de plomb située dans le support indique l'incidence du rayonnement par rapport à la place du dosimètre sur le sujet irradié (de dos ou de face), conduisant si nécessaire à corriger la mesure. Ce dosimètre fut adopté en 1960 par le CEA pour la surveillance de son personnel (figure 9.6).

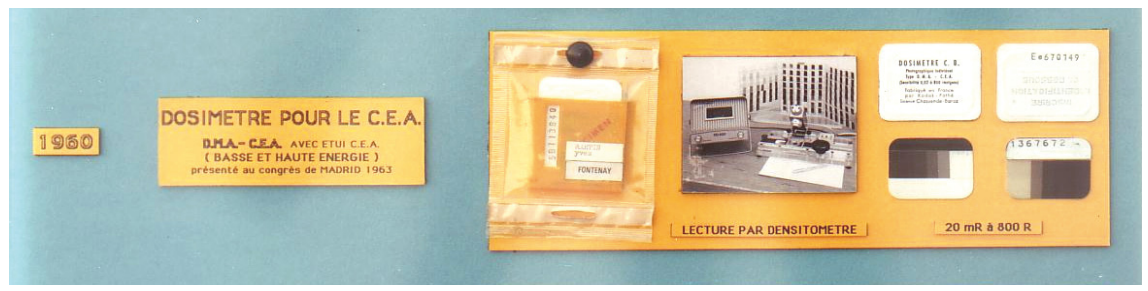

Figure 9.6 - Dosimètre pour le CEA 1960.

En 1967, un nouveau dosimètre guerre « FER 503 » (figure 9.7) est réalisé pour la Marine nationale. Ce dosimètre est une réduction du «DMA-CEA » en format 0 . Il est placé dans un boîtier de plastique et de $1 \mathrm{~mm}$ de plomb «FER 502 » et il enregistre des doses sur une plage de 20 millirads à 800 rads.

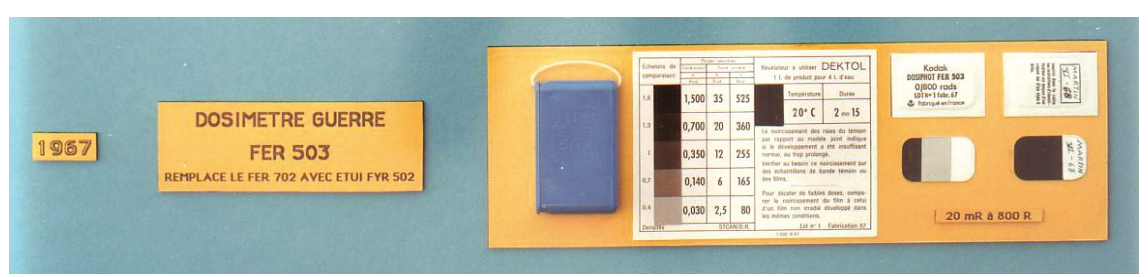

Figure 9.7 - Dosimètre guerre 1967.

En 1970, le dosimètre «DMA-CEA » de format 0 (figure 9.8) est réalisé spécifiquement pour le Service de santé. Ce dosimètre est une réduction du « DMACEA » en format 0 . Il est porté dans un étui spécial étudié par ECERA (2/10 mm de cuivre et $1 \mathrm{~mm}$ de plomb). Il remplacera le DMA-X et le DMA-Co jusqu'en 1974. 


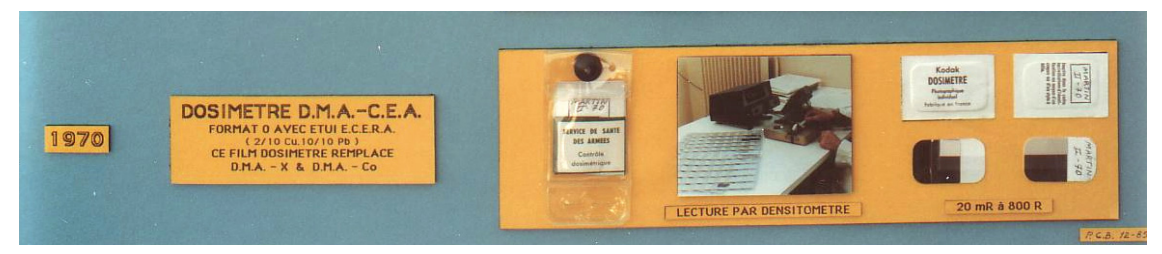

Figure 9.8 - Dosimètre DMA-CEA 1970.

En 1974, les laboratoires de dosimétrie du Service de santé des armées et de l'armement, jusque là indépendants et assurant respectivement la surveillance des établissements médicaux pour le premier et tous les autres centres de la Défense (recherche, contrôle, détection, armées, bâtiments à propulsion nucléaire) pour le second, fusionnent en un seul laboratoire à l'Établissement technique central de l'armement d'Arcueil (ETCA). Cet établissement de la Délégation générale pour l'armement (DGA) avait remplacé le Laboratoire central de l'armement lors de la réorganisation de la DGA. Ce laboratoire de dosimétrie était la principale entité du service Médecine et sécurité du département Détection protection nucléaire ; il restera à Arcueil jusqu'à la fusion du service Médecine et sécurité avec le Service de protection radiologique des armées (SPRA) au $1^{\mathrm{er}}$ janvier 2000.

Le dosimètre choisi pour tous est le DMA-CEA type 1 associé à l'étui PS1, il restera le dosimètre de la défense jusqu'en 1993. Ce dosimètre et son étui ayant été largement présentés dans les chapitres précédents, seules les méthodes d'étalonnages et d'organisation du laboratoire seront développées dans le paragraphe consacré à l'ETCA.

Vers les années 1975-1980, un dosimètre passif fut développé par la DGA spécifiquement pour l'armée de l'air ainsi qu'un lecteur de campagne automatisé : le LYR de dimensions $5 \times 5 \times 0,8 \mathrm{~cm}$. Il était constitué de deux dosimètres, un thermoluminescent (de forme et de dimensions identiques à un fusible) et un verre photoluminescent. La prédose de ce dernier était élevée, de l'ordre de 500 millirems. Il était possible de leur adjoindre une diode pour la dosimétrie des neutrons.

\subsubsection{Les nouveaux dosimètres gamma}

À partir de 1993, la société Kodak décide l'arrêt de la fabrication du dosimètre DMA-CEA type 1 fabriqué et utilisé uniquement en France par l'IPSN (issu du CEA), le LCIE, le CNRS et le ministère de la Défense (ETCA), sa production n'étant plus rentable. Elle propose en remplacement l'utilisation du dosimètre type 2 fabriqué et très utilisé aux États-Unis et au Japon. Le LCIE fait ce choix pour se rapprocher de l'OPRI (Office central de protection contre les rayonnements 
ionisants) qui a succédé au SCPRI, lequel utilisait déjà le Kodak type 2 depuis les années 1970 (voir chapitre 7). Les autres laboratoires portent leur choix vers le dosimètre «personnal monitoring » fabriqué en Belgique par la société Agfa (figure 9.9).

Le format des dosimètres Agfa est celui d'un film dentaire donc inférieur en dimensions au Kodak type 1. Afin de conserver l'emploi des étuis PS 1, ceux cifurent adaptés à l'aide de cales plastiques collées. Leur plage de lecture permet la mesure des doses de $0,1 \mathrm{mSv}$ à $1 \mathrm{~Sv}$ à l'aide de deux émulsions indépendantes et de sensibilités différentes. La mesure de doses plus élevées reste possible soit par décapage soit par un sous-développement. Il ne comporte plus de bande témoin de développement et la mesure du noircissement devient une mesure par transmission (les émulsions sont couchées sur des supports transparents).

Cela eut pour conséquence d'entraîner un changement des lecteurs et une adaptation de tous les supports d'irradiation, de développement et de lecture, sans compter le doublement du nombre des développements pour un même nombre de dosimètres. Il fallut également faire modifier les machines pour le marquage pour les adapter aux nouveaux films.

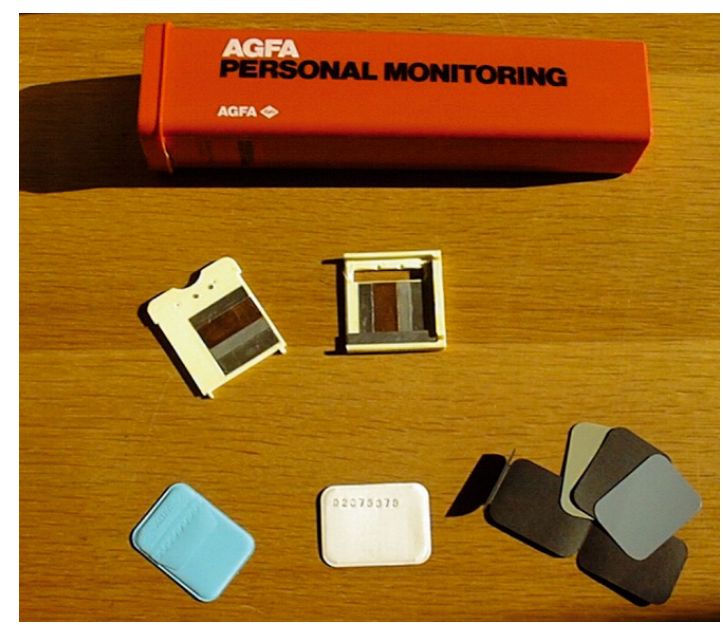

Figure 9.9 - Dosimètre photographique Agfa 1993.

En 2000, le laboratoire de dosimétrie d'Arcueil est rattaché au SPRA revenant ainsi dans le giron du Service de santé des armées, mais les salles de développements des dosimètres restent à Arcueil, ce qui rend l'exploitation difficile. Comme les moyens d'irradiation d'Arcueil sont fermés progressivement à partir de cette période, une partie de l'exploitation est sous-traitée à l'IRSN de 2002 à 2004.

En 2005, toute la dosimétrie de la défense est reprise par le SPRA. La législation n'imposant plus le dosimètre photographique comme seul dosimètre légal, le choix 
du SPRA se porte sur le badge OSL («Optically Stimulated Luminescence ») fabriqué aux États-Unis et commercialisé en France par la société LCIE Landauer (voir chapitres 2 et 10). Afin de conserver le caractère opérationnel de la surveillance des personnels de la défense, le SPRA dispose de lecteurs portables « Microstar » (en mallette de transport) projectables sur le terrain dans le contexte d'opérations extérieures (figure 9.10).

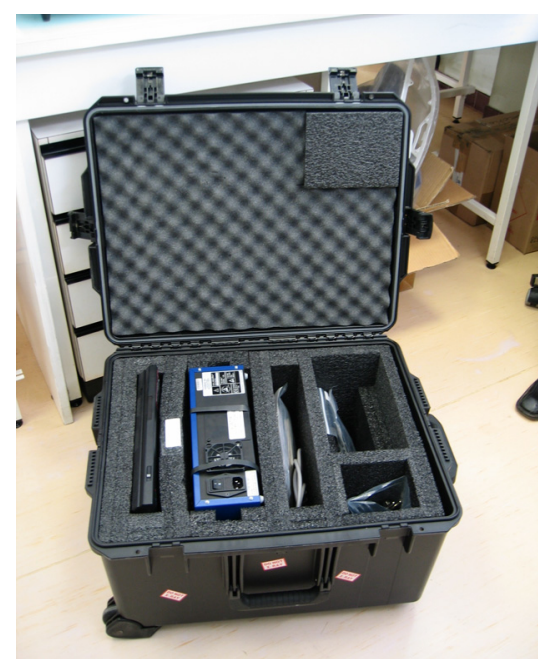

Figure 9.10 - Lecteur portable « Microstar » en mallette de transport.

\subsubsection{Les dosimètres utilisés pour la mesure des neutrons}

La dosimétrie des neutrons a été mise en place avec le début de surveillance des personnels affectés sur les sous-marins nucléaires SNLE en 1970 et pour les personnels des laboratoires de recherche comme le LCA utilisant des neutrons (sources isotopiques américium-béryllium, californium ou accélérateurs Van de Graaf par exemple).

Le seul dosimètre photographique neutron du marché est alors le Kodak NTAA fabriqué aux États-Unis (figure 9.11). Ce dosimètre est associé au dosimètre gamma-X (Kodak type 1 ou Agfa après 1994) dans l'étui PS1. Auparavant, il est inséré dans une pochette d'aluminium à $20 \%$ d'humidité. Il enregistre des doses de $0,40 \mathrm{mSv}$ à $200 \mathrm{mSv}$, cette plage de dose variant avec l'énergie des neutrons (maximale à $14 \mathrm{MeV}$, la réponse chute rapidement jusqu'à $1 \mathrm{MeV}$ ).

Sa sensibilité aux rayonnements gamma peut le rendre inexploitable avec un développement standard (si dose gamma $>10 \mathrm{mSv}$ ) le noircissement empêchant de voir les traces des protons de recul révélées par le développement. 
La mesure de la dose se fait par comptage de traces des protons de recul en comparaison avec un étalonnage, soit lecture à sec avec un objectif de $400 \mu$ pour les doses < à $10 \mathrm{mSv}$ et lecture par immersion avec un objectif de $150 \mu$ pour les doses > à $10 \mathrm{mSv}$. En principe, pour les doses > à $0,80 \mathrm{mSv}$, il faut lire 400 traces pour la surface de référence. Ce comptage au microscope n'était pas mécanisé.

Ce dosimètre sera utilisé par la Défense jusqu'en 2001, date à laquelle la société Kodak arrêta la fabrication.

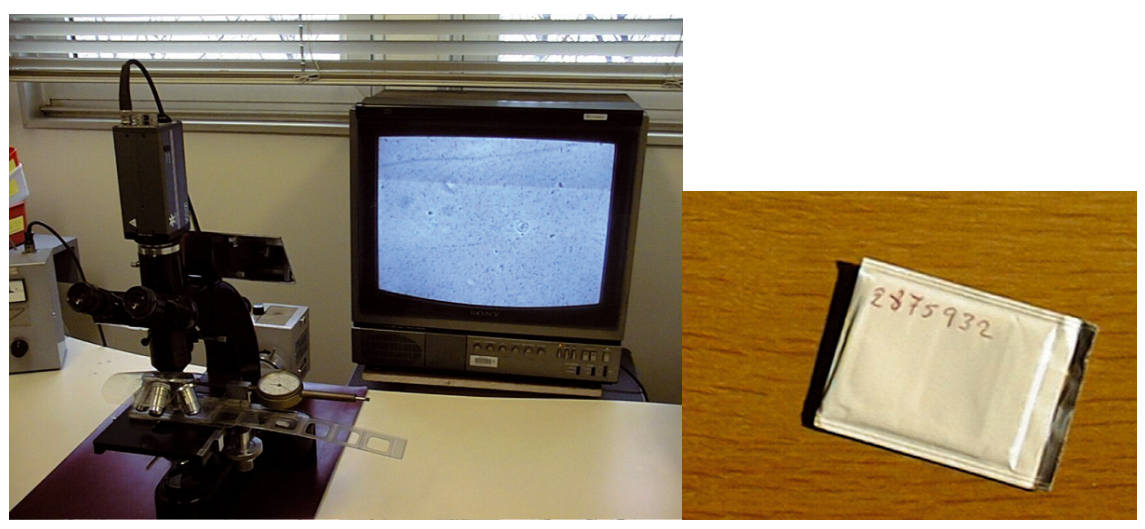

Figure 9.11 - Lecture des dosimètres neutrons NTA.

Il fut alors remplacé par les dosimètres à bulles (figure 9.12) de la société Bubble technology Inc. commercialisés en France par la société Ariès. Bien que considéré comme un dosimètre opérationnel, il sera utilisé par la défense comme dosimètre passif de 2002 à 2004, date à laquelle l'utilisation du dosimètre NP3 fut mise en place.
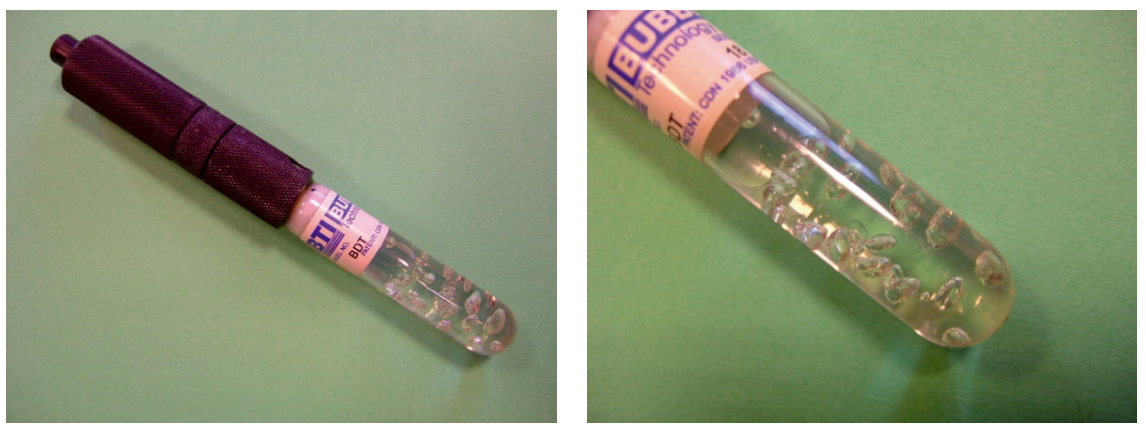

Figure 9.12 - Dosimètre neutrons à bulles. 
Les dosimètres PN3 (figure 9.13), commercialisé en France par la société APVL, est composé d'un polycarbonate de type CR39 dans lequel les neutrons interagissent en créant des protons de recul. Les traces de ces protons sont comptées après développement chimique. Le dosimètre est insensible aux photons et permet la mesure de $0,2 \mathrm{mSv}$ à $200 \mathrm{mSv}$ pour des neutrons d'énergie comprises entre $200 \mathrm{keV}$ et $14 \mathrm{MeV}$. La lecture est automatisée.

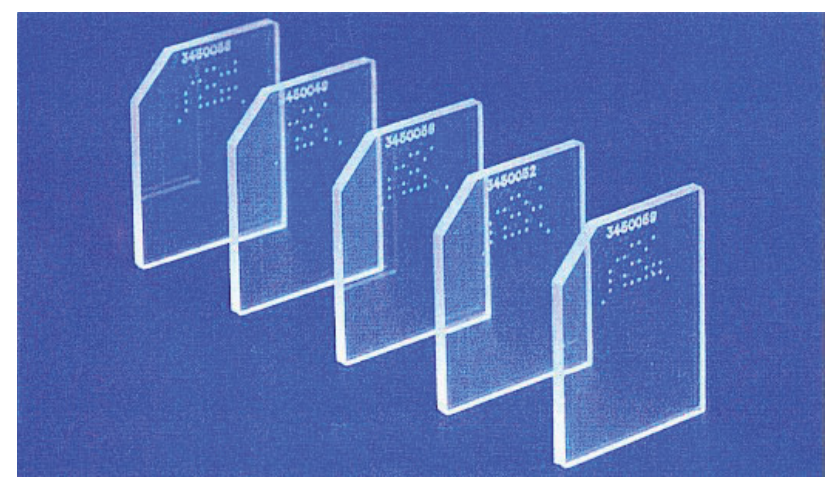

Figure 9.13 - Dosimètre à détection de traces.

Ces différents types de dosimètres ayant fait l'objet de développement dans les chapitres précédents, car également utilisés par d'autres laboratoires, il n'a pas été jugé utile de revenir en détails sur leurs caractéristiques physiques.

L'utilisation de diodes pour la mesure des neutrons demeura au niveau d'études de faisabilité, ou au niveau d'une dosimétrie opérationnelle de complément comme pour le dosimètre LYR. Un lecteur automatique de campagne fut cependant développé.

\subsection{Les différents établissements ayant effectué la dosimétrie (avant 1975)}

Comme il a été dit dans l'introduction, compte tenu de la diversité des organismes concernés et des types de métiers des intervenants, au début de la mise en place de la surveillance dosimétrique plusieurs entités réalisèrent elles-mêmes cette surveillance pour leurs personnels ou la sous-traitèrent à des organismes extérieurs, en particulier le Commissariat à l'énergie atomique. 
Cette pratique ne perdura que quelques années et au fur et à mesure de l'évolution des travaux sous rayonnements et de la restructuration des différents services des armées concernés, il ne subsista que deux services, l'un dépendant du Service de santé des armées, l'autre de la DGA.

Ils fusionnèrent en 1974, absorbant au passage un autre service de la DGA, la Direction des constructions navales de Cherbourg, qui était restée indépendante.

Ces différents établissements seront évoqués brièvement.

\subsubsection{La dosimétrie au Sahara}

La surveillance est réalisée par le Centre saharien d'expérimentation militaire (CSEM) pour les essais atmosphériques puis par le Centre d'expérimentation militaires des oasis (CEMO) pour les essais en galerie.

Cette surveillance concerne les personnels du ministère de la Défense qui ont participé aux expérimentations nucléaires du Sahara, essais aériens puis souterrains.

En ce qui concerne les personnels de la Section technique de l'armée (STA), Groupement arme atomique toujours employés au Fort d'Aubervilliers, la surveillance de 1958 à 1965 est réalisée par le Centre interministériel de décontamination, qui dépend du Centre d'étude atomique de Saclay. Après 1965, une partie de la STA/Y fusionnera avec des établissements d'Arcueil et la surveillance des personnels sera reprise par le Laboratoire central de l'armement qui gardera une antenne à Aubervilliers.

Le dosimètre utilisé par le CSEM et le CEMO est le DMA-CO qui est le premier « dosimètre haute énergie » créé en 1959. Le dosimètre utilisé pour la surveillance des personnels du Fort d'Aubervilliers est le même que celui du CEA.

Les résultats du suivi dosimétrique sont constitués de documents papiers joints au dossier médical des personnels.

Deux exemples de fiches de résultats pour des personnels du Service de santé des armées sont présentés ci-dessous (figure 9.14).

En ce qui concerne les personnels de la STA/Y, les résultats de dosimétrie ont été reportés sur des cahiers qui donnaient mensuellement en milli-Roentgen (mR) la dose organisme entier sous les différents écrans du boîtier (plage nue, sous cadmium et sous étain) et la dose extrémités (poignet) éventuellement. 

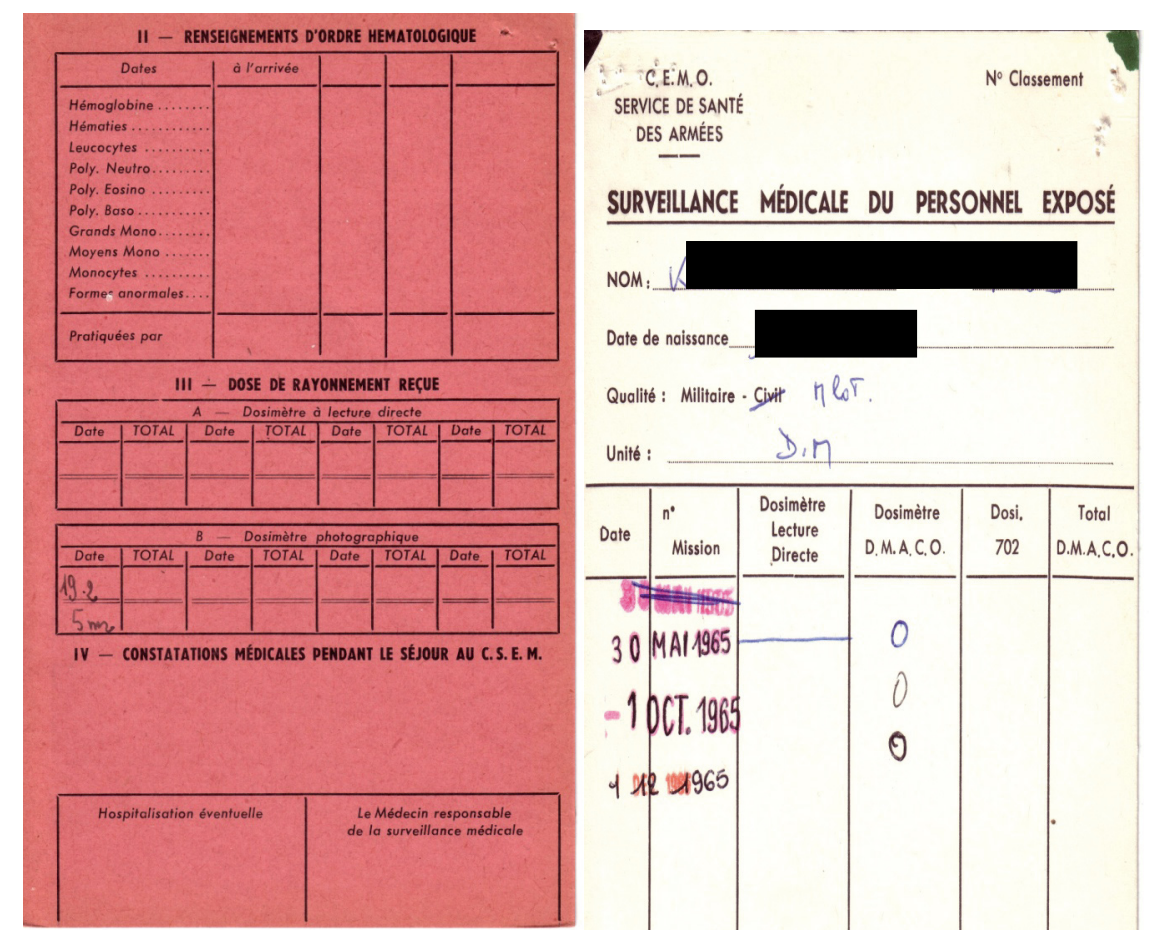

Figure 9.14 - Exemples de fiches de résultats du CSEM et du CEMO.

Tous les résultats évoqués ci-dessus ont été repris dans une base informatique par le Service de protection radiologique des armées.

\subsubsection{La dosimétrie à la Direction des constructions navales $(\mathrm{DCN})$ de Cherbourg et dans quelques autres établissements}

La surveillance des personnels de la DCN de Cherbourg a été réalisée sur place de 1969 à fin 1976 et transférée ensuite à l'ETCA d'Arcueil.

Les résultats ont été repris dans une base informatique par le Service de protection radiologique des armées.

Quelques autres établissements participèrent pour des durées limitées à la surveillance des personnels. En particulier, le département d'études de protection et de décontamination (DEP) de l'établissement technique de Bourges (ETBS) qui 
dépendait d'Arcueil et l'École d'application de la marine pour les études atomiques (EAMEA) à Cherbourg. Ces établissements de formation suivaient en particulier les personnels qui y effectuaient des stages. Les dosimètres étaient les mêmes que ceux utilisés par l'ETCA, qui reprit les résultats dans le fichier général.

\subsubsection{La dosimétrie au Service de santé des armées (SSA)}

La surveillance est réalisée par l'Établissement central d'électroradiologie de l'Armée (ECERA) situé au Fort de Vanves.

Cette surveillance concerne les personnels du Service de santé des armées, exposés aux rayonnements $X$ (radiologue, dentiste, vétérinaire...) ou aux rayonnements de haute énergie (médecine nucléaire, cobalthérapie).

Cette surveillance est exercée de 1957 à décembre 1974, date à laquelle la dosimétrie du SSA est regroupée avec celle du LCA (Laboratoire central de l'Armement), au Fort de Montrouge qui exerce la surveillance de la Marine et des personnels non médicaux du ministère de la Défense. À partir de 1970 a été créé un fichier informatisé.

Les dosimètres utilisés sont soit le DMA-X soit le DMA-Co selon le poste de travail. L'étalonnage des dosimètres gamma est effectué à l'aide d'une source de radium (densité optique correspondante pour les points $50 \mathrm{mR}-100 \mathrm{mR}-200 \mathrm{mR}$ $-400 \mathrm{mR}-800 \mathrm{mR}$ ).

Les résultats exprimés en milirads $(\mathrm{mR})$ sont donnés sous écran de plomb et sans écran.

Le seuil de détection est de $5 \mathrm{mR}$.

Avant 1960, des listings collectifs sont édités chaque mois (figure 9.15).

\subsection{La dosimétrie à l'Établissement technique central de l'Armement}

\subsubsection{Du LCA à l'ETCA}

En 1962, s'installe à Arcueil dans le fort de Montrouge, le Laboratoire central de l'Armement, héritier en ligne plus ou moins directe de l'Atelier de précision créé par le ci-devant Claude-Antoine comte Prieur-Devernois dit Prieur de la Côte d'Or, qui, malgré ses origines, fut élu membre du Comité de salut public, chargé des fabrications d'Armement. Il fit adopter par le Comité l'arrêté du 12 Messidor an II 


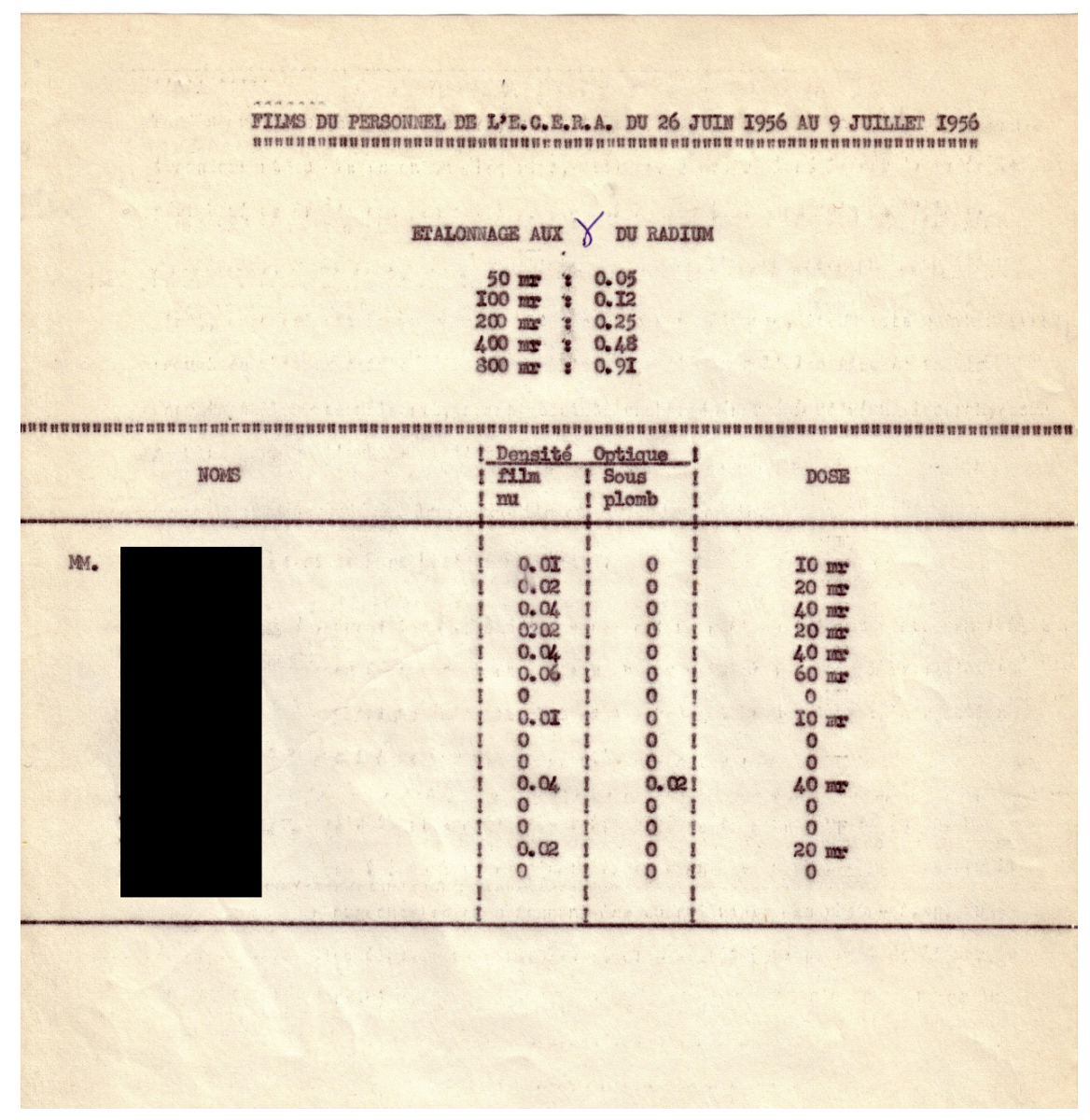

Figure 9.15 - Fiche collective de résultats sur laquelle sont présentes l'identification de l'établissement, le nom de la personne, les densités nue et sous plomb et la dose.

(30 juin 1794) créant « ... dans une maison nationale, un atelier de précision, destiné à la construction des instruments propres à la vérification des projectiles de guerre... » afin d'éviter « ...le malheur tant de fois répété, d'envoyer aux armées des boulets non calibrés...».

Le LCA, tout en conservant son rôle d'atelier de précision, fut chargé d'autres travaux en lien avec les préoccupations du ministère de la défense en ce début des années 1960, et en particulier tous ceux liés aux besoins générés par la politique de dissuasion nucléaire. 
C'est ainsi qu'en 1964, par regroupement de divers service, dont des éléments du STA/Y d'Aubervilliers, le département Détection protection nucléaire vit le jour, doté de moyens d'irradiation nombreux et remarquables, de services de recherche et d'études dans la détection et la métrologie et d'un service « Médecine et sécurité » chargé de la surveillance des personnels travaillant sous rayonnements ionisants, des contrôles de radioprotection des installations de la Défense et de la formation à la radioprotection des personnels concernés.

Ses missions furent effectives à partir de juin 1965 quand tous les moyens et personnels d'Aubervilliers eurent intégré Arcueil.

Il disposait d'un laboratoire de développement, des moyens d'irradiation étalonnés du département, de vastes locaux pour l'évaluation des dosimètres, mais également pour le stockage, la réception et l'envoi des dosimètres, l'archivage des films et des PV de résultats.

Le LCA absorba au cours des années les quelques établissements qui réalisaient leurs propre dosimétrie et surtout fusionna en 1974 avec le Service de santé des Armées quand l'ECERA déménagea du fort de Vanves au fort de Montrouge.

À partir de cette date, à l'exception des Centres d'expérimentation du Pacifique dont la surveillance (à l'exception des établissements médicaux) était assurée par le CEA, toute la surveillance dosimétrique du ministère de la défense fut concentrée à Arcueil.

On s'installa donc dans la durée, passant de préfabriqués à un superbe bâtiment en dur pour le seul service Médecine et sécurité et quand, au cours d'une nouvelle réorganisation de la DGA en 1985, le LCA devint ETCA, seul le nom changea mais pas les missions. Il faudra attendre le $1^{\mathrm{er}}$ janvier 2000 pour un changement de fond, la fusion du Service médecine et sécurité avec le SPRA qui renvoya la dosimétrie au Service de santé des armées et à Clamart dans l'enceinte de l'hôpital d'instruction des armées de Percy.

Tous les établissements de la Défense sont surveillés et les postes de travail sont multiples ; par exemple, pour la marine Nationale, du porte-avions Charles de Gaulle et des sous-marins nucléaires aux infirmeries des plus petits bâtiments des Terres australes ; pour l'Armée de l'Air de la force stratégique, au laboratoire de physique de l'école de l'Air sans oublier les radars; pour le service de santé des hôpitaux des armées aux services de biochimie et de biologie et aux accélérateurs pour la radiothérapie. Les plus spécifiques sont les établissements de la DGA dont l'ETCA lui-même, du déchet radioactif à l'accélérateur de $40 \mathrm{MV}$ en passant par les sources de californium et les lasers de recherche de l'École polytechnique ou le laboratoire d'optique appliquée (LOA) de l'École nationale supérieure des techniques avancées (ENSTA). 
Cette diversité rend le travail du « dosimétriste » intéressant, les personnes qui gèrent l'interprétation savent fort bien détecter les anomalies parfois visibles sur les films et les spécificités des types d'exposition en fonction des différentes sources de rayonnements renvoyant aux nombreux métiers.

\subsubsection{Les dosimètres utilisés et les techniques d'exploitation}

Le film Kodak «Type 1 associé à l'étui PS1 a été utilisé jusqu'en 1993 date à laquelle Kodak en a arrêté la fabrication devenue non rentable car seule la France utilisait encore ce dosimètre.

Il possède une zone de marquage pour inscrire le nom du porteur et/ou le numéro du dosimètre : le laboratoire dispose de machines à marquer semi-automatiques, anciennes (elles avaient été faite spécialement pour le service de santé dès le début de l'exploitation des dosimètres) mais extrêmement fiables, bien qu'un peu bruyantes.

Dès réception des lots de films, le marquage est lancé de manière à pouvoir faire face aux expéditions mensuelles ou trimestrielles et aux demandes urgentes. Les numéros étant incrémentés, aucun numéro n'a été utilisé deux fois - on a ainsi pu fêter le $2000000^{\mathrm{e}}$ film, quelque temps avant l'arrêt de la fabrication.

Le film présente un témoin de développement et trois émulsions de sensibilité différentes permettant une gamme de mesure de 20 mrem à 800 rems avec des zones de recoupement :

- première émulsion : ultra-rapide de 20 mrem à $1 \mathrm{rem}(0,2 \mathrm{mSv}$ à $10 \mathrm{mSv})$;

- deuxième émulsion : rapide de 0,8 rem à $25 \mathrm{rems}(0,8 \mathrm{mSv}$ à $250 \mathrm{mSv})$;

- troisième émulsion : lente de 20 rems à 800 rems $(200 \mathrm{mSv}$ à $8 \mathrm{~Sv})$.

Pendant toute la durée de validité des lots, un contrôle mensuel vérifie qu'il n'y a pas saturation de la densité optique jusqu'à 1000 rems $(10 \mathrm{~Sv})$.

Des contrôles qualités propres à augmenter la fiabilité de la mesure se sont mis en place progressivement.

\section{Contrôle à la réception}

Une seule commande par an est passée à Kodak, dans la mesure du possible en un seul lot ayant la date de péremption la plus retardée possible et au moins supérieure à un an.

À réception du lot, un contrôle de l'homogénéité du voile de fond se fait par prélèvement d'un film par boîte identifiée. Ce fut rare mais quelques lots furent retournés en totalité pour voile de fond trop élevé. 
Le lot et la date de péremption sont gravés sur le film et son emballage à la fabrication.

L'évolution du voile de fond est suivie mensuellement.

\section{Étalonnages}

Les trois émulsions sont étalonnées par irradiation à différentes énergies : gammas du cobalt 60 et du césium 137, RX de $50 \mathrm{kV}$ à $300 \mathrm{kV}$ avec filtrations correspondant aux normes AFNOR en séries large ou étroite.

Pour chaque dose, dix dosimètres sont exposés à chaque valeur retenue (doses très rapprochées pour l'émulsion ultrarapide et au niveau des zones de recouvrement des émulsions).

Un étalonnage mensuel de contrôle de l'émulsion la plus sensible est réalisé par irradiation au seul cobalt, mais sur quelques valeurs et avec trois dosimètres par point.

Le LCA (puis l'ETCA) disposent à cette époque de tout un ensemble de sources isotopiques de différentes activités (de moins de $10 \mathrm{mCi}$ à $60000 \mathrm{Ci}$ ) et de tubes à RX (Philips Majorix) (figure 9.16) fonctionnant de 50 à $300 \mathrm{kV}$ avec fenêtres de sortie béryllium ou aluminium; des jeux de filtres permettent d'obtenir les énergies souhaitées avec une résolution plus ou moins large (Séries AFNOR).

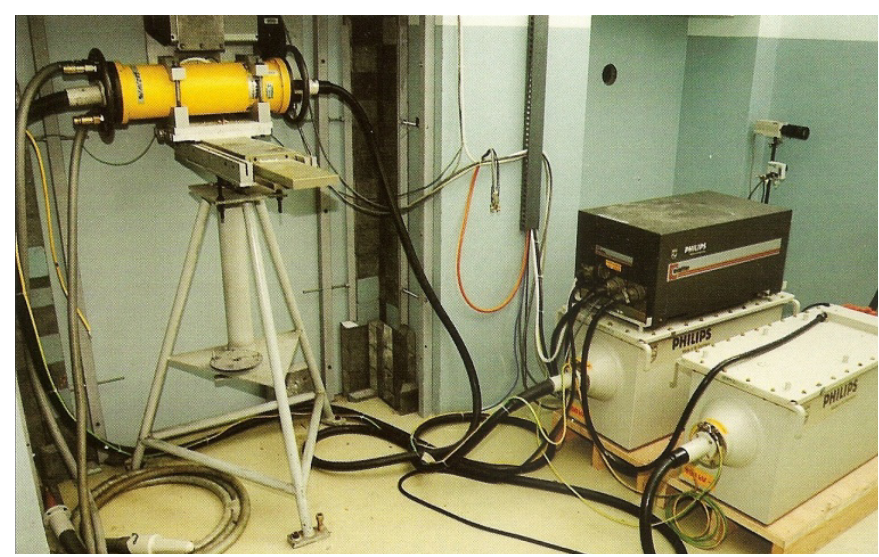

Figure 9.16 - Tubes à RX et leur alimentation. 


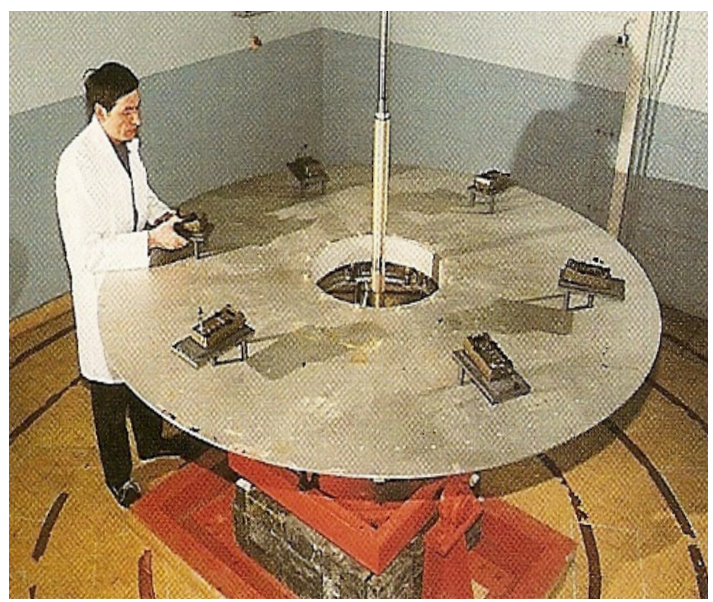

Figure 9.17 - Salle d'étalonnage des dosimètres.

La salle de dosimétrie, de volume cubique et de grande dimensions pour minimiser le diffusé, est équipée d'un conteneur à barillet comportant six sources d'activités étagées. La source sélectionnée est extraite par un système pneumatique dans un guide central. Des plateaux tournants en aluminium (homogénéité de l'irradiation), interchangeables, reçoivent des supports en plexiglas adaptés aux détecteurs à irradier. Les dosimètres (films + étui PS1) sont disposés en couronne à $50 \mathrm{~cm}$ ou $1,20 \mathrm{~m}$ de la source pour les irradiations - ces deux distances ayant été étalonnées pour l'ensemble des sources disponibles (figures 9.17 et 9.18).

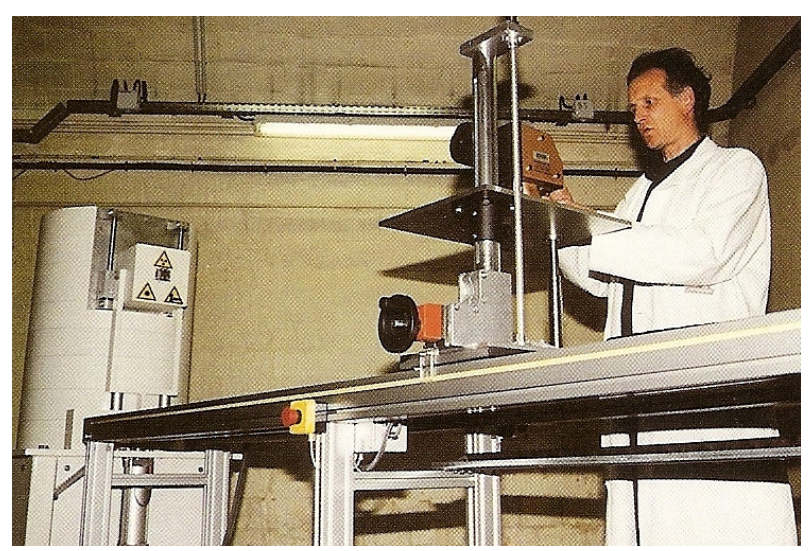

Figure 9.18 - Salle d'étalonnage, faisceau collimaté. 
Le LCA-ETCA est laboratoire secondaire du Bureau national de métrologie et fut même un temps laboratoire primaire, pour les RX, du Bureau international des poids et mesures.

Pour les RX, il dispose d'une chambre absolue et la mesure se fait par la méthode du taux de dérive, où l'on compense la charge de capacités étalonnées (figure 9.19). Bien entendu la mesure est en Rœntgen et les courants mesurés sont de l'ordre de $10^{-15}$ A. Pour la mesure des plus hautes énergies, on utilise des chambres Shonka équivalent air.

Des intercomparaisons régulières sont organisées avec le Laboratoire de métrologie des rayonnements ionisants (LMRI).

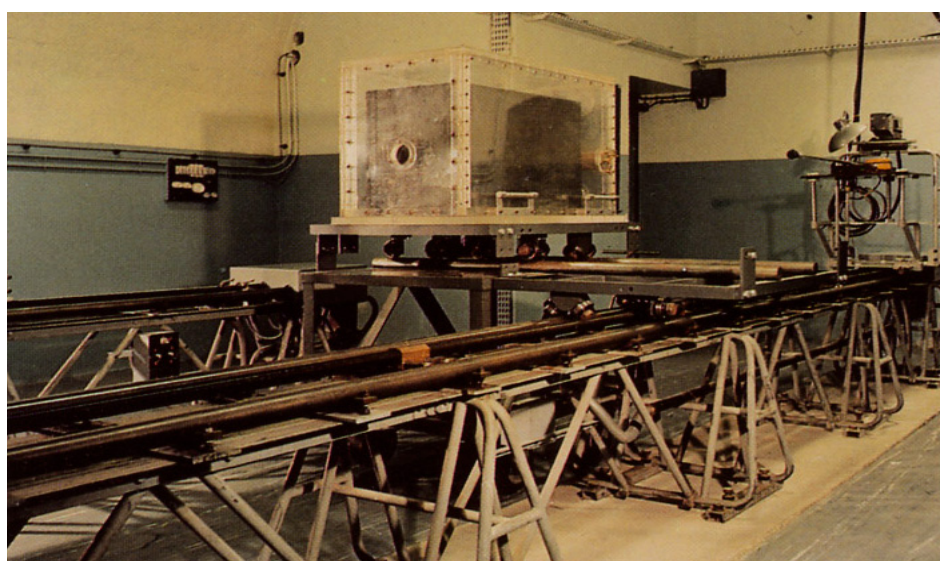

Figure 9.19 - Chambre absolue.

Auparavant, l'ECERA utilisait des sources de radium pour ses étalonnages ; à Aubervilliers, le laboratoire de la STA/Y disposait de sources de cobalt 60 et de césium 137 de différentes activités et de tubes à RX. Leur étalonnage se faisait à l'aide d'une chambre de transfert par comparaison avec des sources de radium 226 dont la masse (et donc l'activité) était connue. Les calibrations des sources d'irradiation pour les étalonnages des dosimètres de la DCN et de l'EAMEA étaient réalisés avec des chambres de transfert de l'ETCA.

Après irradiation et attente de $24 \mathrm{~h}$, les films étalonnés sont développés. La lecture de la densité optique sous toutes les plages de l'étui PS1 est réalisée (double lecture par deux personnes différentes) à l'aide d'un densitomètre à réflexion de 
marque Macbeth (figure 9.20). Un étalon de noircissements de référence est inséré avant chaque série de mesures pour étalonner le densitomètre. Un densitomètre est réservé aux seuls étalonnages.

Pour optimiser la mesure, les films pour la lecture sont positionnés dans un système mécanique qui permet l'avance automatique d'une plage à l'autre. Cet adaptateur a l'avantage de permettre un repositionnement précis non seulement pour les étalonnages mais pour tout type de relecture.

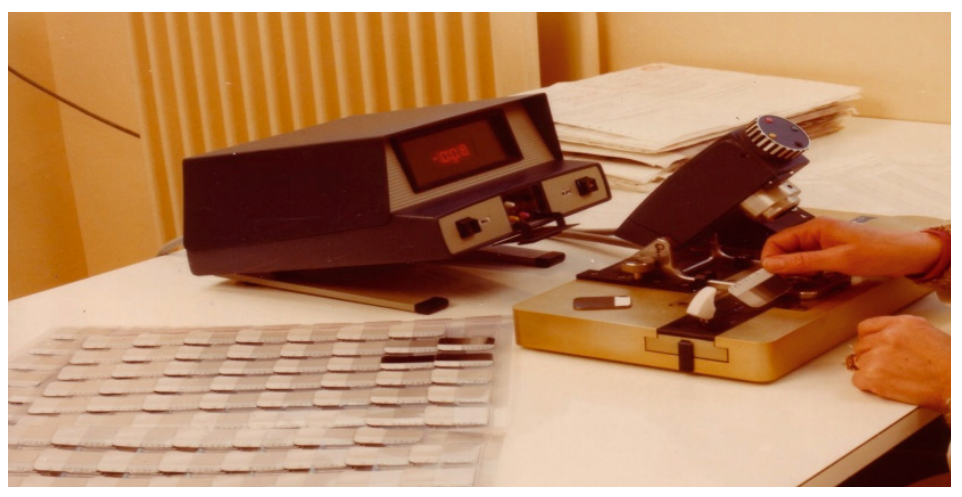

Figure 9.20 - Densitomètre Macbeth et support de lecture.

- Courbes d'étalonnage correspondant aux trois émulsions

Pour chaque lot de dosimètres, des étalonnages sont réalisés afin de tracer les courbes densité/dose pour les trois émulsions (voir figures 9.21, 9.22 et 9.23). Sur ces courbes tracées à la main figurent en abscisses les doses et en ordonnées les densités optiques. Sont également notés le lot de dosimètres et la date d'étalonnage.

Les dosimètres exposés sont développés et lus et la dose est attribuée en fonction des courbes d'étalonnage, selon le type de rayonnements auquel le dosimètre a été soumis. Cette estimation, manuelle au commencement évoluera avec l'informatisation progressive du laboratoire (calcul automatique de la dose par interpolation à partir de la fonction mathématique représentant la courbe d'étalonnage).

- La salle de développement (figure 9.24)

Un sas, peint en noir avec bandes blanches de guidage, à double chicane et sans porte, donne accès à la salle de développement. Il n'y a qu'au cinéma que l'on rit quand un panier rempli de films est percuté par une porte qui s'ouvre dans le noir ; les portes battantes initiales furent donc bannies après quelques déboires. 


\section{J. BRIAND-CHAMPLONG et al.}

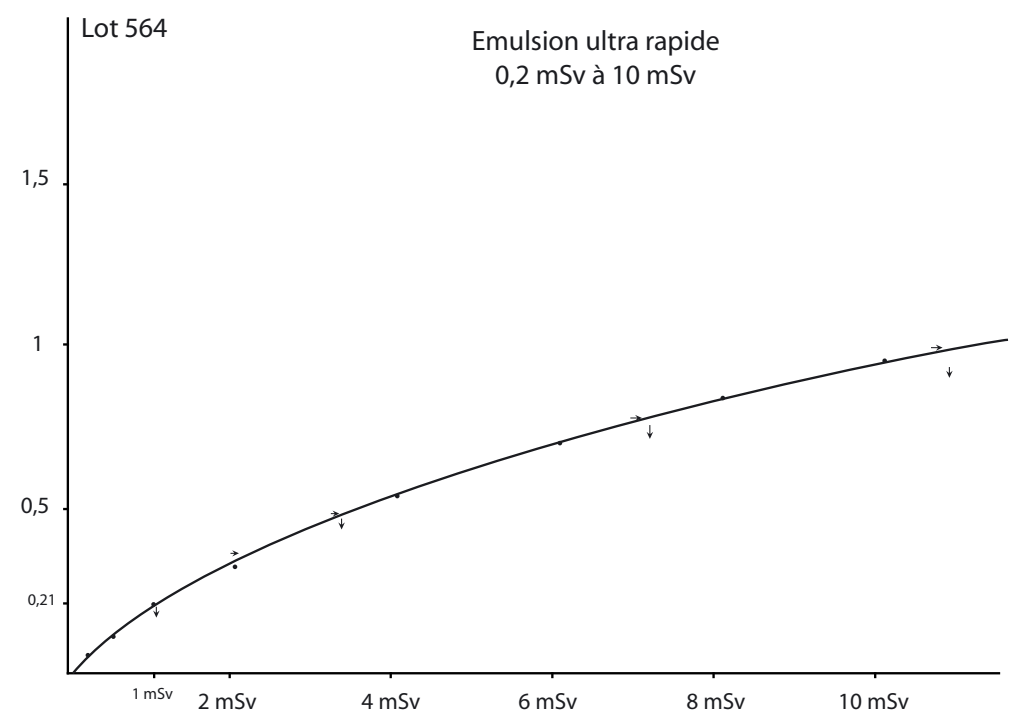

Figure 9.21 - Émulsion ultrarapide 0,20 à $10 \mathrm{mSv}$.

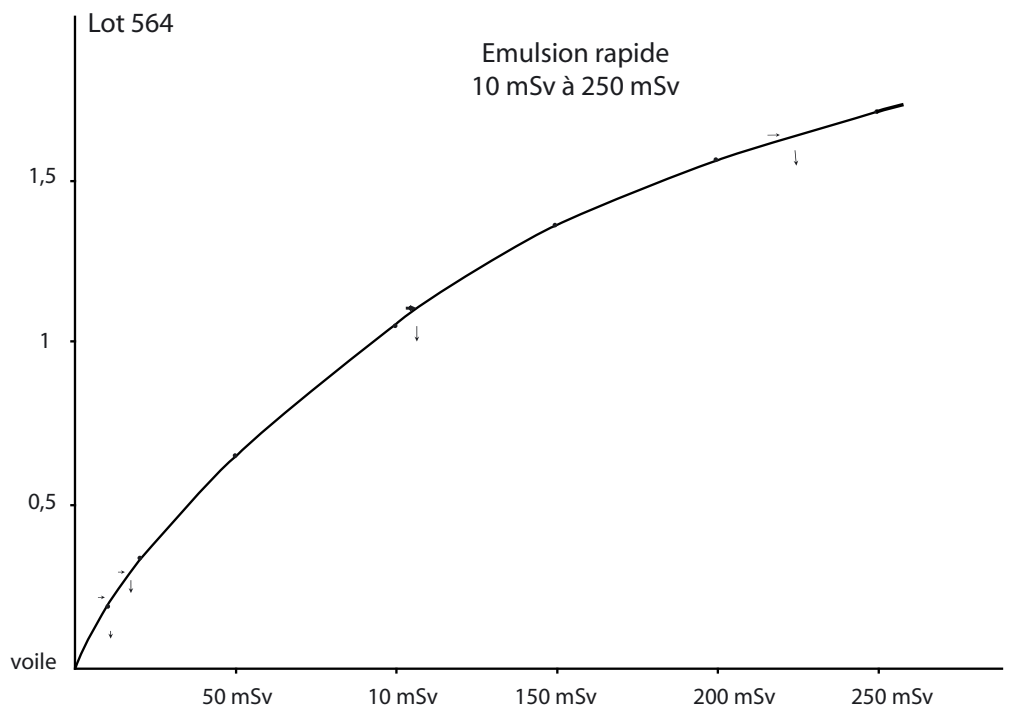

Figure 9.22 - Émulsion rapide $10 \mathrm{mSv}$ à $250 \mathrm{mSv}$. 


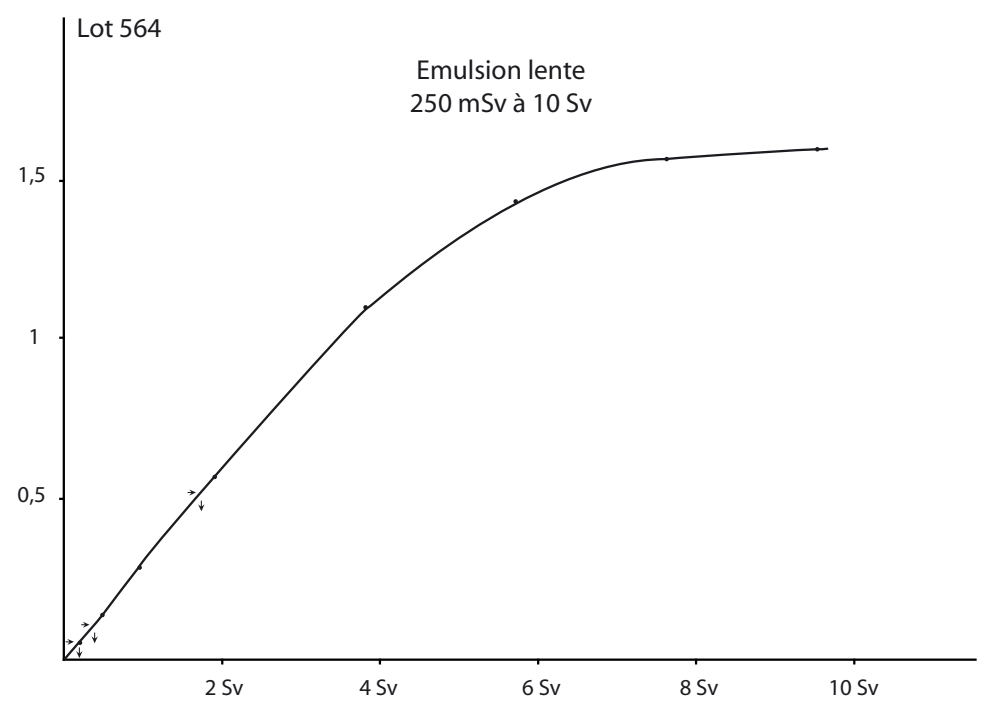

Figure 9.23 - Émulsion lente $250 \mathrm{mSv}$ à $10 \mathrm{~Sv}$.

Des lumières inactiniques, dont l'intensité est bridée pour éviter de voiler les émulsions, permettent de travailler dans de conditions satisfaisantes dans la salle de développement climatisée à $20^{\circ} \mathrm{C}$. Tout d'abord les films sont extraits de leur enveloppe après découpe par percussion (appareillage automatique développé en interne). Ils sont placés dans l'ordre de la numérotation dans des paniers. Dès qu'un panier est rempli il est placé dans un cadre opaque en attente du développement.

Les interrupteurs des lampes inactiniques sont à hauteur normale, les autres situés à $1,80 \mathrm{~m}$ pour éviter tout éclairement intempestif.

Les bains de développement sont changés chaque semaine le vendredi afin de se stabiliser durant le week-end pour permettre les développements dès le lundi matin.

Révélateur gamma :

La préparation commence par celle du concentré : dissoudre peu à peu une boîte de Dektol Kodak dans 4 litres d'eau distillée à une température de $30^{\circ} \mathrm{C}$, à l'aide d'un agitateur. Compléter à 5 litres avec de l'eau distillée.

Pour le développement la cuve de révélateur est constituée de 4 litres de révélateur concentré et de 8 litres d'eau distillée. 
Après chaque développement, on ajoute dans la cuve 500 cc de révélateur dilué dans les mêmes proportions.

Le révélateur est changé après 5 développements.

Bain d'arrêt gamma :

Verser 300 cc d'acide acétique (1N) dans 10 litres d'eau distillée et compléter à 15 litres avec de l'eau distillée.

Fixateur gamma :

Dissoudre 3 litres de fixateur concentré AL4 Kodak dans 12 litres d'eau distillée.

Révélateur neutron :

La préparation commence par celle du concentré : dissoudre peu à peu une boîte de $\mathrm{D} 19 \mathrm{~b}$ Kodak dans 4 litres d'eau distillée à une température de $30^{\circ} \mathrm{C}$, à l'aide d'un agitateur. Compléter à 5 litres avec de l'au distillée.

Pour le développement, la cuve de révélateur est constituée de 3 litres de révélateur concentré et de 6 litres d'eau distillée.

Bain d'arrêt neutron :

Le bain est constitué de 180 cc d'acide acétique (1N) versés dans 7 litres d'eau distillée et étendus à 9 litres avec de l'eau distillée.

Fixateur neutron :

Pour ce bain, dissoudre $3 \mathrm{~kg}$ d'hyposulfite de sodium puis $300 \mathrm{~g}$ de sulfite de sodium dans 4 litres d'eau distillée, puis compléter à 10 litres avec de l'eau distillée. Ajuster le pH à 6,8 soit environ 30 cc d'acide acétique $(1 \mathrm{~N})$.

Bain mouillant neutron :

Ce bain est constitué de 1,5 cc de mouillant Photo-Flo Kodak dilué dans 9 litres d'eau distillée.

\section{Les contrôles à l'exploitation}

- Expédition des dosimètres

Après prise en compte des mouvements de personnels ou d'ambiance (cessation ou création de surveillance dosimétrique), l'expédition du mois suivant peut-être programmée. L'ordinateur attribue les numéros de films (gamma-X et neutron) à partir du dernier numéro de la dotation du mois précédent, à chaque personnel et à 


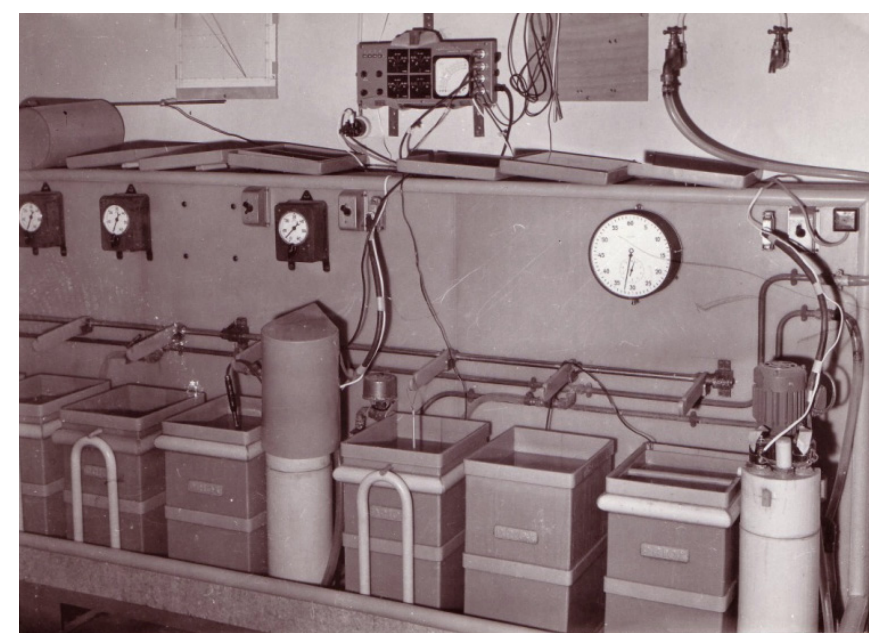

Figure 9.24 - La salle de développement (version 1).

chaque ambiance, plus un certain nombre de numéros disponibles pour les films non attribués, ceci pour chaque établissement.

Parallèlement à l'édition des bordereaux d'envoi, tous les films à expédier sont marqués, par encreur pour les films gamma-X ou par estampage pour les NTA-A (gravure du numéro dans le support plastique de l'émulsion). Les NTA-A sont ensuite conditionnés ; ils sont glissés dans une pochette étanche constitué par un papier aluminisé et plastifié portant le même numéro que l'émulsion. Avant d'être soudées à chaud, ces pochettes sont mises à dessécher en boîte à gants pendant 24 heures afin d'obtenir un taux d'humidité inférieur à $20 \%$.

Ils sont ensuite associés au film gamma de même numéro et glissés ensemble dans un étui PS1 puis dans une pochette soudée transparente.

- Retour des dosimètres

Au retour de chaque colis, un comptage précis du nombre de films retournés est effectué ; il doit correspondre au nombre indiqué par l'établissement.

Si tout est conforme, les films sont envoyés en salle de développement.

Pour le contrôle dans chaque développement, des films témoins sont insérés. (En cas de difficultés ils permettent de définir le coefficient correcteur à appliquer à l'ensemble des films du développement). 
Ces témoins comportent :

- un témoin «blanc» (voile de fond);

- un témoin irradié à 100 mrem $(1 \mathrm{mSv})$;

- un témoin irradié à 5 rem $(50 \mathrm{mSv})$;

placés dans chaque panier de films pour contrôler également l'homogénéité du développement. Lors du passage au film Agfa, ces valeurs furent modifiées pour tenir compte du changement de sensibilité des émulsions.

La préparation des développements se fait par paquet de 40 films +témoins, $(20+$ témoins pour les Agfa) avec édition d'une feuille de suivi précisant les établissements et le panier dans lequel se trouvent les films et le nombre de films si le panier n'est pas complet.

À la mise en panier des films contrôle, on s'assure qu'il ne reste pas de place vide (elle indiquerait soit que deux films ont été mis dans la même case, soit qu'un film a été jeté avec les emballages). On a aussi la possibilité de compter les pochettes d'où l'on a extrait les films ; avant de commencer le panier suivant, les pochettes sont vidées par une trappe dans un réceptacle.

Ces enveloppes de films seront conservées un mois.

Un développement comprend au maximum 5 paniers de 80 films, un couvercle spécial est positionné au-dessus pour bloquer les films et éviter qu'ils ne quittent leur logement.

Le contrôle des températures et du bullage à l'azote (pression 150 millibars pour les gamma, 350 millibars pour les neutrons) afin d'obtenir une plus grande homogénéité du développement en forçant la circulation du produit entre les films, est vérifié avant l'insertion des paniers dans les bains.

Une double minuterie est mise en route; la minuterie principale est préprogrammée au temps normal de développement pour éviter une erreur de programmation, la seconde sert de secours en cas de panne électrique.

Le développement comprend six phases successives :

\section{Développement gamma}

- bain révélateur Kodak : 2 minutes 15 secondes ;

- bain d'arrêt : 1 minute ;

- un premier bain fixateur : 15 minutes ;

- un second bain de fixateur : 15 minutes.

- le double bain est plus efficace pour augmenter la qualité de la conservation du film dans le temps.

- bain de lavage à l'eau courante tempérée : 15 minutes ; 
- second bain de lavage à l'eau courante tempérée :15 minutes.

Après développement, les films dans leur panier sont placés dans une étuve réglée à $40^{\circ} \mathrm{C}$, pour un temps de séchage de six heures.

\section{Développement neutron}

- bain révélateur : 30 minutes ;

- bain d'arrêt : 5 minutes ;

- un premier bain fixateur : 30 minutes ;

- bain de lavage à l'eau courante tempérée : 30 minutes ;

- bain mouillant : 10 minutes.

Après développement, les films dans leur panier sont placés dans une étuve réglée à $40^{\circ} \mathrm{C}$, pour un temps de séchage de six heures.

Dans la mesure du possible, les films sont développés dans les 24 heures qui suivent leur arrivée. Entre les établissements un ordre de priorité existe : sousmarins (transmission des résultats des ambiances dans les 48 heures, DCN, ETBS, SSA...

Le lendemain du développement, les paniers sont vidés et les films sont placés dans des passe-vues et regroupés avec le bordereau d'expédition qui contient toutes les informations sur les porteurs ou les ambiances.

Après lecture des densités optiques (sous chaque plage de l'étui PS1) ou comptage des traces des NTA-A, les valeurs trouvées sont interprétées par comparaison avec les courbes d'étalonnages et l'on procède à l'édition des procèsverbaux des résultats.

Les valeurs des seuils d'enregistrement étaient de $20 \mathrm{mrem}(0,2 \mathrm{mSv})$ pour les films gammas, $10 \mathrm{mrem}(0,1 \mathrm{mSv})$ pour les $\mathrm{X}$ et de $50 \mathrm{mrem}(0,5 \mathrm{mSv})$ pour les neutrons.

L'ensemble des documents, feuilles de lecture des densités optiques, courbes d'étalonnage, lecture des témoins a été archivé pour servir de preuve de qualité en cas de contentieux, de même pour les films eux-mêmes (jusqu'en 2004) ainsi qu'un exemplaire des PV envoyés.

\section{Anomalies}

- si le nombre de films est différent de celui attendu;

- si le film présente une anomalie (insolation, humidité, détérioration);

- si le film présente une image anormale (contamination, image d'objet);

la pochette du film est recherchée (les pochettes sont gardées dans des boîtes correspondant au jour de développement pendant une semaine - d'une manière générale les pochettes sont conservées jusqu'à l'édition complète des résultats). 


\section{Dose d'alerte}

Lors du classement en passe-vues, tout film présentant une dose de 500 mrem ( $5 \mathrm{mSv}$ ) est détecté par une comparaison visuelle avec le témoin correspondant. Si ce film est un organisme entier, la lecture de la densité optique est effectuée et la dose exacte calculée. Un message est adressé le jour même à l'établissement et la $\mathrm{PCR}$ est jointe téléphoniquement. Cette valeur de $5 \mathrm{mSv}$ fut ramenée à $2 \mathrm{mSv}$ après 2003, en raison de l'abaissement des limites réglementaires.

\section{Développement d'urgence}

En cas d'accident radiologique dans un établissement ou si la PCR ou le médecin du travail suspecte un incident ou un dépassement de limite, il est possible de faire développer le dosimètre en urgence : une estimation est faite visuellement à la fin du développement et le résultat définitif est donné en 6 heures.

\section{Changement de film}

En 1993, le passage vers le film Agfa remplaçant le Kodak type 1 entraîne des modifications.

Le film Agfa comportant deux émulsions sur deux supports différents, la mise en panier a été changée, une bande pour l'émulsion rapide, une pour l'émulsion lente. Le nombre des développements a donc doublé, aussi en 1998 la salle a été automatisée par un robot (figure 9.25).

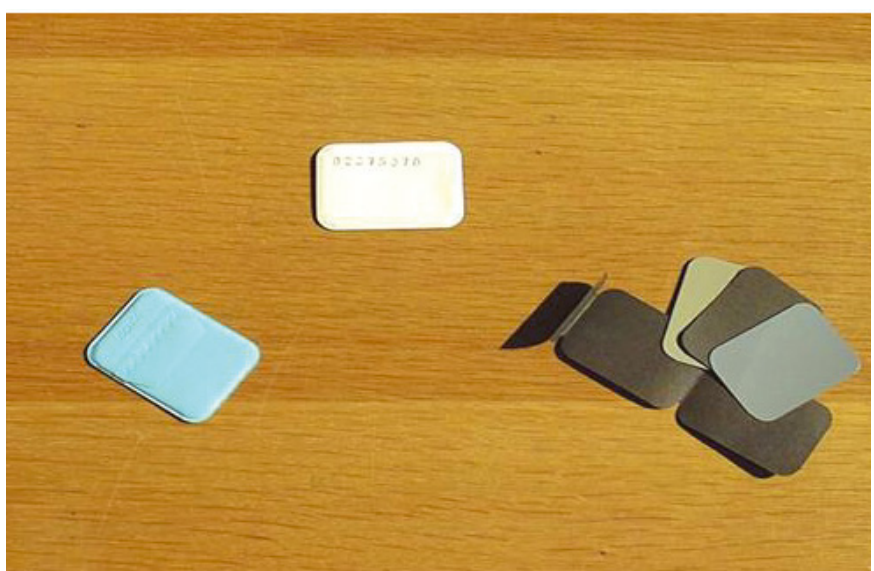

Figure 9.25 - Le dosimètre Agfa comprenant une enveloppe plastique blanche sur la face externe et verte au dos, deux films (émulsion lente et émulsion rapide) entourés de cavaliers en papier noir. 
Les deux émulsions de sensibilités différentes permettent une gamme de mesure de $0,20 \mathrm{~Sv}$ à $1 \mathrm{~Sv}$ avec zone de recoupement sur deux supports différents.

- première émulsion : $0,20 \mathrm{mSv}$ à $50 \mathrm{mSv}$;

- deuxième émulsion : $30 \mathrm{mSv}$ à $1 \mathrm{~Sv}$.

Les émulsions du film Agfa sont couchées de part et d'autre du support d'acétate de cellulose.

La lecture maximale de la densité optique est de 5. La dose maximale mesurable est donc bien inférieure à celle du film Kodak type1. Deux solutions ont été retenues pour y remédier :

- sous-développement des dosimètres avec un étalonnage limité dans le cas où le dépassement possible est annoncé ;

- décapage d'une des deux couches sensibles (cas où le dépassement est fortuit). Ces deux solutions permettent de mesurer des doses jusqu'à $10 \mathrm{~Sv}$.

Un aménagement du système obligea à adapter les machines existantes.

Le marquage a été réalisé par percussion sur des supports en caoutchouc dur, qui ont permis de graver le numéro sur les deux émulsions sans percer l'emballage étanche.

Le lecteur par réflexion Macbeth est remplacé par un lecteur par transmission de marque XRite.

Les bains de développement sont toujours renouvelés chaque semaine, mais un week-end ne suffit pas pour atteindre la stabilisation (développement optimal) ; il faut attendre une dizaine de développements. Deux méthodes furent utilisées pour pallier ce problème : tout d'abord plusieurs développements de films non utilisés, méthode abandonnée assez vite car coûteuse en temps, puis le remplacement partiel du bain de révélateur (après filtration du bain précédent) fut retenue.

Les durées de passage dans le révélateur et le fixateur furent adaptées en fonction des informations données par le fabricant.

Les grands principes de préparations et de travail restent en place tout en s'orientant vers une automatisation et une informatisation du travail.

\subsubsection{Le fichier et les documents d'exploitation}

Au commencement de la surveillance de l'exposition, la gestion de l'enregistrement des doses reçues est manuelle sur les fiches et procès verbaux de résultats tapés à la machine (avec carbone pour l'exemplaire d'archivage). 


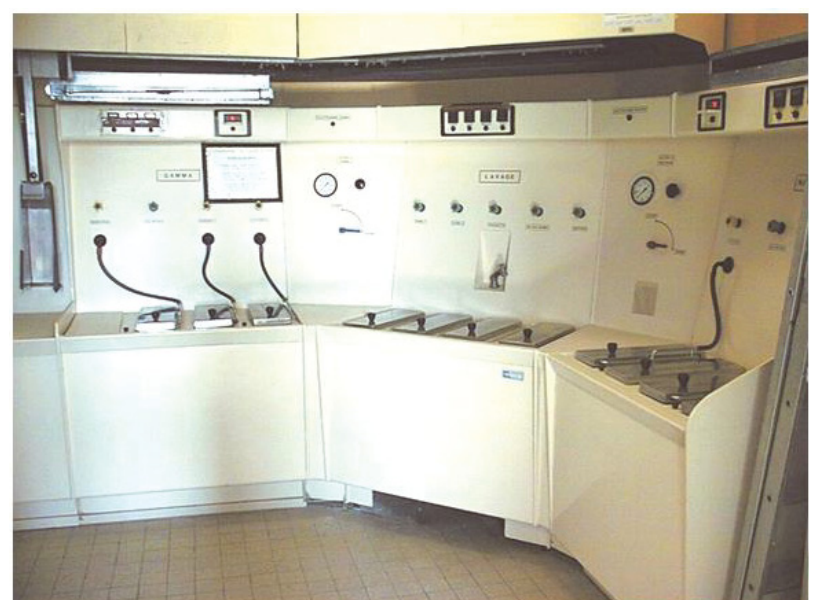

Figure 9.26 - La salle de développement (version 2).

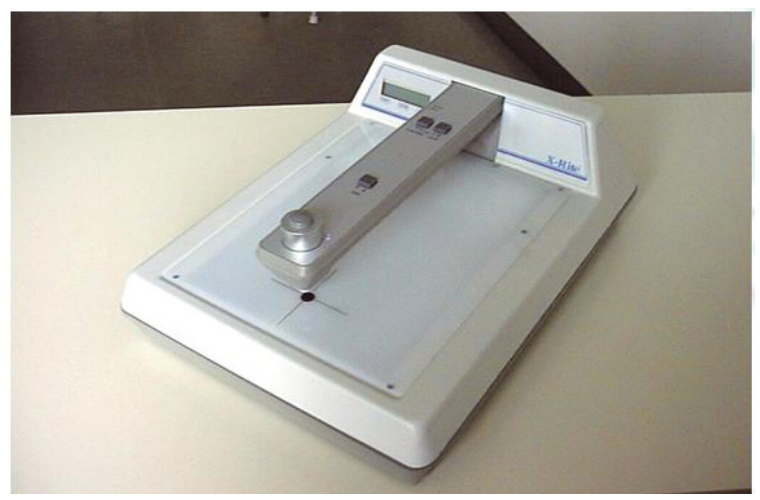

Figure 9.27 - Densitomètre à transmission X Rite 361.

En 1970, le LCA réalise une base de données informatique en utilisant les moyens lourds du centre de calcul d'Arcueil (UNIVAC). Deux cartes perforées de 90 caractères chacune synthétisent les informations concernant une personne.

S'il semble qu'en France Dupont et Durand soient les noms les plus courants, dans le fichier du ministère de la défense la palme revient aux Lefèvre (ou Lefebvre) et aux Martin et les noms d'origine bretonne sont très nombreux.

Chaque personne entrant dans le fichier reçoit un numéro de dosimétrie à cinq chiffres qui est unique ; elle le conserve même si elle change d'établissement. 
Les établissements sont référencés avec un numéro à quatre chiffres (par exemple : 4XXX pour la marine, 5XXX pour l'armée de l'Air, 1XXX pour les établissements DGA...). Il existait aussi un numéro en $9 \mathrm{XXX}$ pour de très petits établissements atypiques et lointains et pour les «bonnes œuvres » de la Défense, des infirmeries ou des associations humanitaires qui étaient surveillées gratuitement. Plus de trois cents établissements sont ainsi répertoriés.

Le métier de la personne est décrit par un code emploi (intérêt pour les statistiques et surtout pour expliciter les incidents révélés par le film (spécificité du type de rayonnement attendu par exemple). Plus d'une centaine de codes emplois est utilisée.

Le numéro INSEE à 13 chiffres sert pour éviter tout risque de doublon ou d'erreur de personne et le fichier a été déclaré à la CNIL (mais plus tardivement). Il n'apparait pas dans les PV.

À partir de 1975, le service de santé est également intégré dans la base de données.

Pour le recueil des informations concernant les personnes ou les ambiances de travail, les demandes de surveillance doivent préciser également le type de film (gamma X ou gamma associé à un film neutron) et, si nécessaire, la fourniture d'un dosimètre d'extrémités.

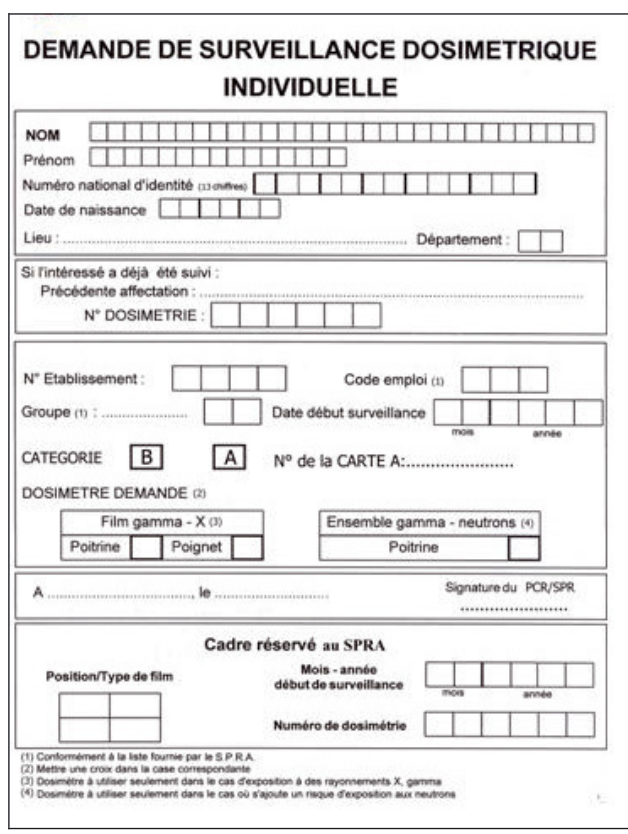

Nom et Prénoms

Date et lieu de naissance RNIPP

Code Etablissement et sous-groupe éventuel dans l'établissement Type de film : G-X ou G-N Position : OE (organisme entier) et si besoin un film EXT (extrémités) en complément

Code Emploi

Date de début de surveillance

Les renseignements sur l'ambiance sont :

Libellé du poste

Etablissement et groupe

Type de film : G-X ou G-N

Position Z (zone) ou T (témoin)

Date de début de surveillance 
J. BRIAND-CHAMPLONG et al.

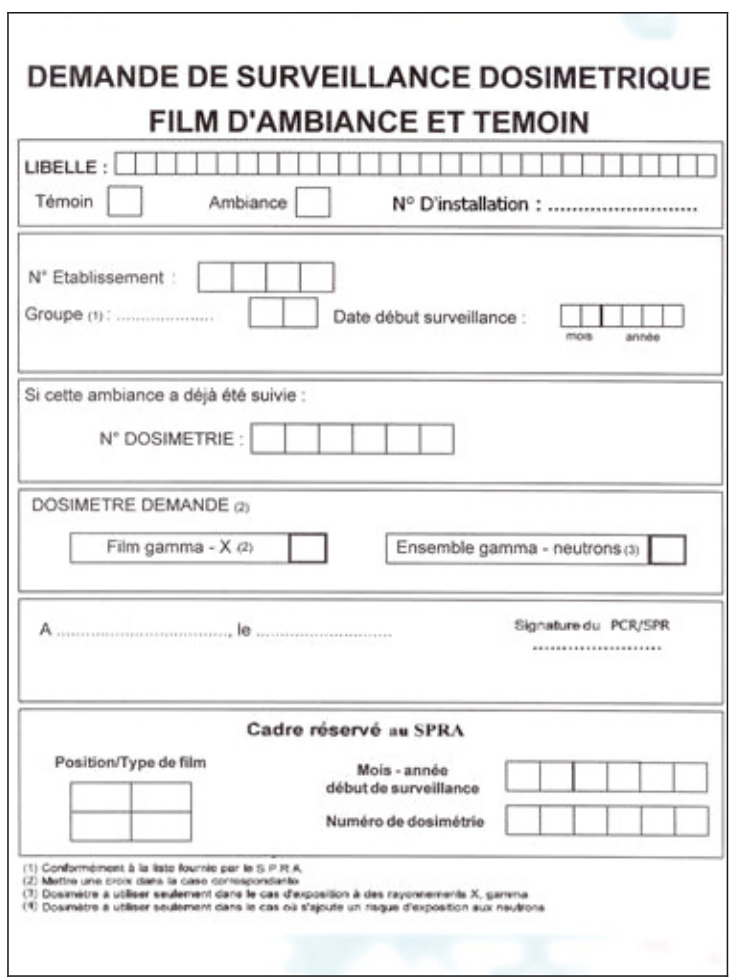

Pour les bordereaux d'envoi et de retour accompagnant les dosimètres, deux types de listing existent.

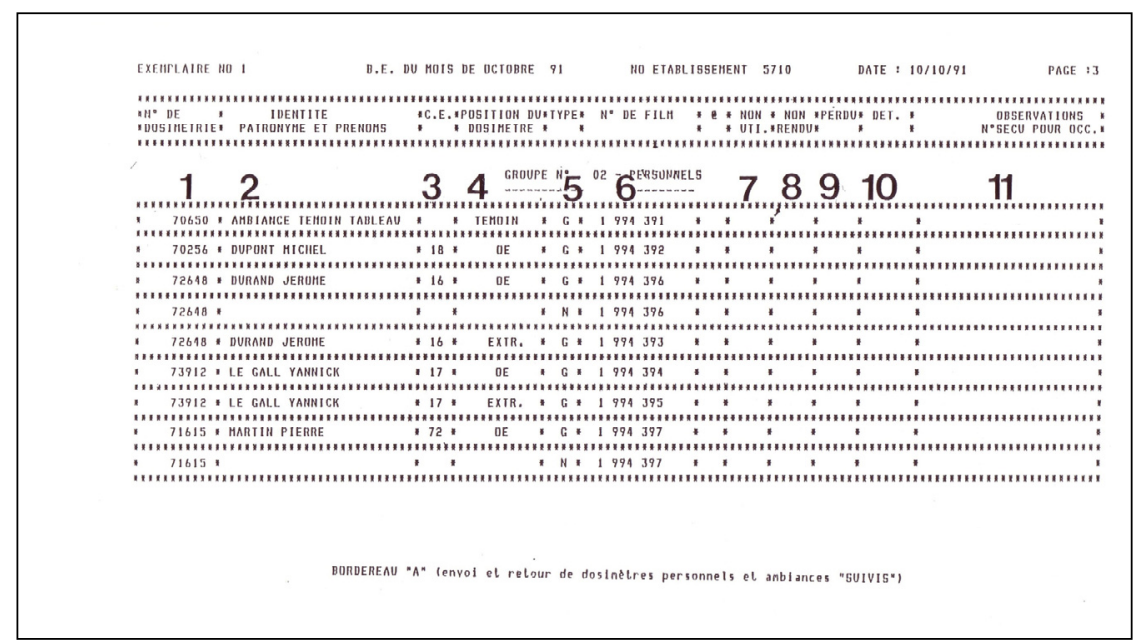


Listing des « suivis » :

1 et 2 : Attribution ( $\mathrm{n}^{\circ}$ de dosimétrie et nom de la personne ou libellé ambiance).

3 : Code emploi pour la personne.

4 : Position du film (OE ou EXT).

5 : Type de film (G-X ou G-N).

$6: \mathrm{N}^{\circ} \mathrm{du}$ film.

(la demande de surveillance est à l'origine de ces informations).

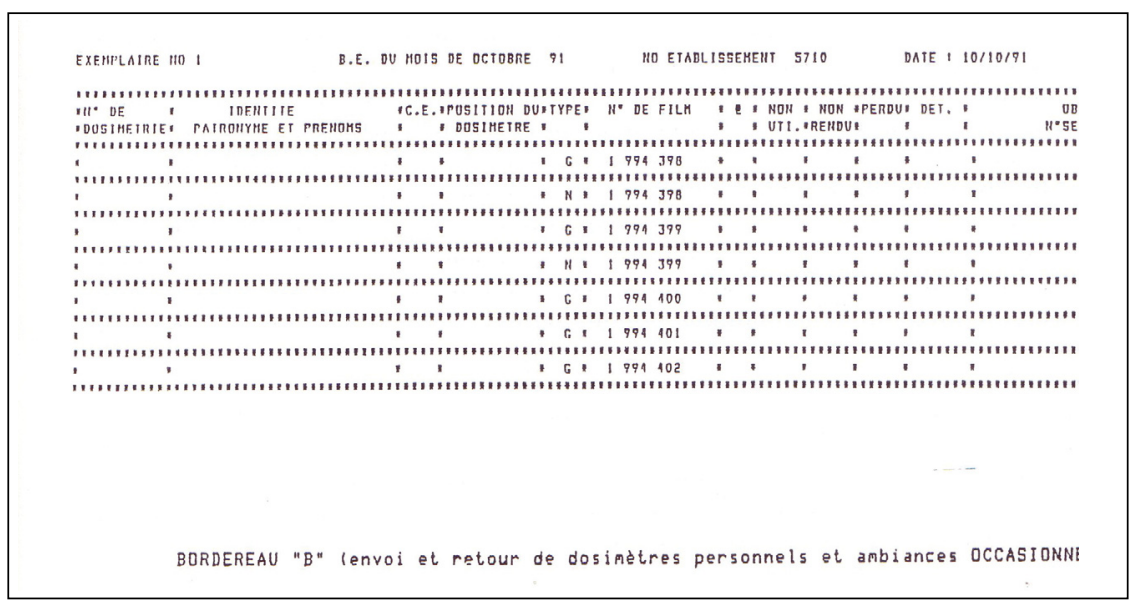

Listing des « occasionnels » : Type de film et $\mathrm{N}^{\circ} \mathrm{du}$ film.

Les autres informations sont remplies sur place par l'établissement.

Il existe aussi un troisième type de listing plus rare heureusement : celui concernant la demande de développement de dosimètres rendus en retard.

L'informatique ayant évolué très rapidement, le service Médecine et sécurité s'est doté en 1987 d'un ordinateur IN 500 puis IN8500 de Intertechnique pour s'affranchir des moyens de calcul extérieur. L'archivage se fait sur bandes magnétiques dont un double est conservé dans une armoire ignifugée.

Depuis 1998, la gestion, l'édition des bordereaux d'envoi accompagnant les dosimètres et l'expédition des bordereaux de résultats se font sur simple PC.

Les bordereaux étaient imprimés en quatre exemplaires, un était archivé, un était adressé au Service de protection radiologique des armées, les deux autres étaient 
destinés à l'établissement, l'un pour le médecin du travail ou de prévention, l'autre pour la personne compétente en radioprotection ou la personne chargée de la surveillance des personnels dans l'établissement.

- Quelques chiffres

La surveillance dosimétrique par films photographiques dans la défense a représenté entre 11000 et 15000 dosimètres gamma ou X par mois ainsi que 800 à 2000 dosimètres neutrons NTA pour un peu plus de 300 établissements.

La capacité maximale en routine de développement représentait 2400 films par jour pour 6 développements complets. Il fallut passer à 8 développements par jour au moment du changement pour le film Agfa avec deux équipes en décalé.

Le fichier actuel représente près de 190000 numéros de dosimétrie (personnes et ambiances).

- Le bêtisier

Comme tout bon laboratoire de dosimétrie photographique, l'ETCA possédait son «imagier ». À côté des très sérieuses comparaisons X et gamma, on pouvait observer les accidents du type trou d'épingle ou d'agrafes, les passages en machine à laver (pour blanchiment sans doute) - seule une température de $90{ }^{\circ} \mathrm{C}$ (courante hélas avec les blouses en coton) empêchait l'interprétation. Très rarement, une radiographie d'objet métallique - ciseaux ou réglet dans une poche apparaissait.

Ce qui était fréquent - tant que le service militaire fut maintenu - était l'irradiation volontaire, en fin d'incorporation, soit quatre fois par an, de dosimètres dans le faisceau direct des tubes dentaires. Les dentistes facétieux étaient persuadés que les dosimètres n'étaient pas développés et s'amusaient ainsi une ou deux fois avant la fin de leur service. La découverte évidente de la supercherie entraînait cependant courrier, enquête, recherche de l'individu et rapport à rédiger et à classer sans suite. La plaisanterie cessa lorsqu'un obstiné récidiviste se retrouva en observation durant un mois dans un lit d'hôpital, ainsi puni par un médecin chef excédé ; le bouche à oreilles fit le reste à Libourne...

\subsection{Aujourd'hui et demain}

L'arrêté Défense du 9 juillet 1980 (Journal officiel du 29 juillet 1980) dans son article 17 des dispositions particulières, chargeait l'ETCA de la surveillance de l'exposition : «L'établissement technique central de l'armement est chargé pour l'ensemble du ministère de la Défense, d'exécuter la dosimétrie physique individuelle et d'ambiance : le service Médecine et sécurité de cet établissement tient à jour le fichier dosimétrique des personnels pour le compte du service de protection radiologique des armées et le met à sa disposition. » 
Aujourd'hui, c'est le SPRA qui en à la charge complète - surveillance et fichier ; (cf. l'arrêté du 25 janvier 2005, paru au Journal officiel du 2 février 2005, relatif aux missions du Service de protection radiologique des armées).

Conformément aux exigences relatives aux laboratoires assurant la surveillance de l'exposition des personnels, le SPRA est accrédité par le COFRAC depuis fin 2004 et a reçu un agrément délivré par l'Autorité de sûreté nucléaire.

Les documents propres à la surveillance dosimétrique sont publiés dans le guide relatif aux dispositions communes en matière de protection radiologique du personnel du ministère de la défense.

La dosimétrie par badge OSL Landauer a remplacé le film photographique et les détecteurs polymères NP3 l'émulsion nucléaire NTA-A.

Les étalonnages sont sous-traités à l'IRSN et les enregistrements de la surveillance - presque exclusivement trimestrielle compte tenu des niveaux de dose enregistrés et des résultats des études de poste - sont transférés dans la base nationale SISERI.

\section{Conclusion}

La boucle est donc bouclée : partie du Service de santé des armées, la dosimétrie est retournée au Service de santé des armées. Tout aussi logique et rassurant, la surveillance de l'exposition des personnels de Défense, civils et militaires, s'est fondue dans la surveillance générale française des personnels travaillant sous rayonnements, puisqu'en temps de paix rien ne différencie le militaire du civil et que la réglementation s'applique à tous et pour tous.

Il n'empêche qu'il y eut une histoire de la dosimétrie dans la Défense et que tous ceux qui participèrent à cette aventure et vécurent ces transformations s'en souviennent avec joie et parfois nostalgie, mais ont été vraiment intéressés de la faire revivre à la demande d'Alain Biau qu'ils remercient de les avoir associés à sa publication.

Il n'est pas possible bien entendu, de citer tous les acteurs de cette longue démarche, mais avec Martine Guilland qui fut leur fidèle élève nous dédions spécialement ce modeste témoignage à madame Kientzel, aujourd'hui disparue et quifut l'assistante du pharmacien général Chassende-Barozet à Pierre ChassendeBaroz, fils du pharmacien général, qui fit bénéficier de son expérience et de son inventivité le laboratoire de dosimétrie photographique et mit au point de nombreux moyens propres à assurer la qualité de cette surveillance et bien entendu à toutes celles et tous ceux qui participèrent à cette aventure et la conduisirent à l'accréditation. 